\title{
Multi-scale analysis for wave problems with disparate propagation speeds
}

\author{
Mihaï BOSTAN *
}

(February 28, 2022)

\begin{abstract}
The subject matter of this work concerns the asymptotic behavior of the wave problems, when the propagation speed in one direction is much larger than in the other directions. We establish weak and strong convergence results. We appeal to homogenization arguments, based on average operators with respect to unitary groups.
\end{abstract}

Keywords: Average operators, Ergodic means, Unitary groups, Homogenization.

AMS classification: 35L05, 37A10

\section{Introduction}

We focus on the behavior of the solutions for wave equations whose propagation speed becomes very large along some direction. We consider the problem

$$
\begin{gathered}
\partial_{t}^{2} u^{\varepsilon}-\operatorname{div}_{y}\left(D(y) \nabla_{y} u^{\varepsilon}\right)-\frac{1}{\varepsilon^{2}} \operatorname{div}_{y}\left(b(y) \otimes b(y) \nabla_{y} u^{\varepsilon}\right)=0, \quad(t, y) \in \mathbb{R}_{+} \times \mathbb{R}^{m} \\
u^{\varepsilon}(0, y)=u_{\text {in }}^{\varepsilon}(y), \quad \partial_{t} u^{\varepsilon}(0, y)=\dot{u}_{\text {in }}^{\varepsilon}(y), \quad y \in \mathbb{R}^{m}
\end{gathered}
$$

where $D(y) \in \mathcal{M}_{m}(\mathbb{R})$ and $b(y) \in \mathbb{R}^{m}$ are given matrix and vector fields on $\mathbb{R}^{m}$. We use the notation $\xi \otimes \eta$ for the matrix whose entry $(i, j)$ is $\xi_{i} \eta_{j}$, where $\xi, \eta \in \mathbb{R}^{m}$, and $A: B$ for $\operatorname{trace}\left({ }^{t} A B\right)=\sum_{i=1}^{m} \sum_{j=1}^{m} A_{j i} B_{j i}$, with $A, B \in \mathcal{M}_{m}(\mathbb{R})$. The matrix field $D$ is assumed symmetric non-negative, such that $D+b \otimes b$ is positive definite. We concentrate on the behavior of the family $\left(u^{\varepsilon}\right)_{\varepsilon}$ for $0<\varepsilon \leq 1$, in which case $\left(D+\frac{1}{\varepsilon^{2}} b \otimes b\right)_{0<\varepsilon<1}$ remain positive definite. This study is motivated by the numerical simulation of highly anisotropic wave problems. Indeed, the explicit methods require small time steps, through the CFL stability condition $\Delta t \sim \varepsilon|\Delta y|$. Therefore suitable

*Aix Marseille Université, CNRS, Centrale Marseille, Institut de Mathématiques de Marseille, UMR 7373, Château Gombert 39 rue F. Joliot Curie, 13453 Marseille FRANCE. E-mail : mihai.bostan@univ-amu.fr 
methods have been proposed $[7,12,10,13]$, see also $[9,11,1,16,17]$ for elliptic and parabolic problems.

We consider variational solutions for (1), (2). We introduce a weighted Sobolev space $H_{P}^{1}$ see $(8)$ and define the bounded symmetric bilinear form

$$
a^{\varepsilon}(u, v)=\int_{\mathbb{R}^{m}} D(y) \nabla u \cdot \nabla v \mathrm{~d} y+\frac{1}{\varepsilon^{2}} \int_{\mathbb{R}^{m}}(b \cdot \nabla u)(b \cdot \nabla v) \mathrm{d} y, \quad u, v \in H_{P}^{1} .
$$

By standard results cf. [14], for any $\varepsilon \in] 0,1]$ and $\left(u_{\text {in }}^{\varepsilon}, \dot{u}_{\text {in }}^{\varepsilon}\right) \in H_{P}^{1} \times L^{2}\left(\mathbb{R}^{m}\right)$, there is a unique solution $u^{\varepsilon} \in C\left(\mathbb{R}_{+} ; H_{P}^{1}\right), \partial_{t} u^{\varepsilon} \in C\left(\mathbb{R}_{+} ; L^{2}\left(\mathbb{R}^{m}\right)\right)$ for

$$
u^{\varepsilon}(0)=u_{\text {in }}^{\varepsilon}, \partial_{t} u^{\varepsilon}(0)=\dot{u}_{\text {in }}^{\varepsilon}, \quad \frac{\mathrm{d}}{\mathrm{d} t} \int_{\mathbb{R}^{m}} \partial_{t} u^{\varepsilon} v(y) \mathrm{d} y+a^{\varepsilon}\left(u^{\varepsilon}(t), v\right)=0 \text { in } \mathcal{D}^{\prime}\left(\mathbb{R}_{+}\right), v \in H_{P}^{1}
$$

where $\mathcal{D}\left(\mathbb{R}_{+}\right)$is the set of infinitely many times differentiable functions, compactly supported in $\mathbb{R}_{+}$and $\mathcal{D}^{\prime}\left(\mathbb{R}_{+}\right)$stands for the distributions on $\mathcal{D}\left(\mathbb{R}_{+}\right)$. We are interested on the asymptotic behavior of the family $\left(u^{\varepsilon}\right)_{\varepsilon}$, when $\varepsilon \searrow 0$. We prove that the limit problem corresponds to a wave operator as well, and identify the matrix field associated to it. The identification of the limit model relies on averaging along the characteristic flow of the vector field $b$

$$
\frac{\mathrm{d} Y}{\mathrm{~d} s}=b(Y(s ; y)), \quad(s, y) \in \mathbb{R} \times \mathbb{R}^{m}, \quad Y(0 ; y)=y, \quad y \in \mathbb{R}^{m} .
$$

For example, the orthogonal projection of any function $u \in L^{2}\left(\mathbb{R}^{m}\right)$ over the subspace of $L^{2}$ functions which are left invariant with respect to the flow of $b$, writes

$$
\operatorname{Proj}_{\text {ker } b \cdot \nabla_{y}} u=\lim _{S \rightarrow+\infty} \frac{1}{S} \int_{0}^{S} u \circ Y(s ; \cdot) \mathrm{d} s, \text { strongly in } L^{2}\left(\mathbb{R}^{m}\right) .
$$

The above orthogonal projection appears as the average along the characteristic flow of $b$. Similarly, under suitable hypotheses, we introduce the average of a matrix field $A=A(y)$ by

$$
\langle A\rangle:=\lim _{S \rightarrow+\infty} \frac{1}{S} \int_{0}^{S} \partial Y(-s ; Y(s ; \cdot)) A(Y(s ; \cdot)){ }^{t} \partial Y(-s ; Y(s ; \cdot)) \mathrm{d} s .
$$

The previous average operator will enter the limit model and we prove the following convergence results, see Theorems 4.1, 4.2 for exact statements including all the hypotheses on the vector field $b$ and the matrix field $D$. Here the notation $\mathcal{T}$ stands for the operator $u \mapsto \operatorname{div}_{y}(u b), u \in \operatorname{dom} \mathcal{T}$.

Theorem 1.1

Assume that the initial conditions satisfy

$$
\lim _{\varepsilon \searrow 0} u_{\text {in }}^{\varepsilon}=u_{\text {in }} \quad \text { weakly in } H_{P}^{1}, \quad \lim _{\varepsilon \searrow 0} \dot{u}_{\text {in }}^{\varepsilon}=\dot{u}_{\text {in }} \quad \text { weakly in } L^{2}\left(\mathbb{R}^{m}\right)
$$

and

$$
\sup _{0<\varepsilon \leq 1} \frac{\left|b \cdot \nabla u_{\text {in }}^{\varepsilon}\right|_{L^{2}\left(\mathbb{R}^{m}\right)}}{\varepsilon}<+\infty
$$


Then $\left(u^{\varepsilon}\right)_{\varepsilon},\left(\partial_{t} u^{\varepsilon}\right)_{\varepsilon}$ converge weakly $\star$ in $L^{\infty}\left([0, T], H_{P}^{1}\right), L^{\infty}\left([0, T] ; L^{2}\left(\mathbb{R}^{m}\right)\right)$ respectively, $T \in \mathbb{R}_{+}$, toward the solution $\left(u, \partial_{t} u\right) \in L_{\mathrm{loc}}^{\infty}\left(\mathbb{R}_{+} ; H_{P}^{1} \cap \operatorname{ker} \mathcal{T}\right) \times L_{\mathrm{loc}}^{\infty}\left(\mathbb{R}_{+} ; \operatorname{ker} \mathcal{T}\right)$ of the problem

$$
\frac{\mathrm{d}}{\mathrm{d} t} \int_{\mathbb{R}^{m}} \partial_{t} u v \mathrm{~d} y+\int_{\mathbb{R}^{m}}\langle D\rangle(y) \nabla u \cdot \nabla v \mathrm{~d} y=0 \quad \text { in } \mathcal{D}^{\prime}\left(\mathbb{R}_{+}\right), v \in H_{P}^{1}
$$

with $\left(u(0), \partial_{t} u(0)\right)=\left(u_{\text {in }},\left\langle\dot{u}_{\text {in }}\right\rangle\right)$.

When the initial conditions are well prepared, the above weak convergences become strong.

\section{Theorem 1.2}

Assume that the initial conditions satisfy

$$
\begin{aligned}
\lim _{\varepsilon \searrow 0} u_{\mathrm{in}}^{\varepsilon}= & u_{\mathrm{in}} \text { strongly in } H_{P}^{1}, \lim _{\varepsilon \searrow 0} \frac{b \cdot \nabla u_{\mathrm{in}}^{\varepsilon}}{\varepsilon}=0 \text { strongly in } L^{2}\left(\mathbb{R}^{m}\right) \\
& \lim _{\varepsilon \searrow 0} \dot{u}_{\mathrm{in}}^{\varepsilon}=\dot{u}_{\mathrm{in}} \text { strongly in } L^{2}\left(\mathbb{R}^{m}\right), \quad b \cdot \nabla \dot{u}_{\mathrm{in}}=0 .
\end{aligned}
$$

Then we have the strong convergences

$$
\begin{gathered}
\lim _{\varepsilon \searrow 0} u^{\varepsilon}=u \quad \text { in } L_{\mathrm{loc}}^{\infty}\left(\mathbb{R}_{+} ; L^{2}\left(\mathbb{R}^{m}\right)\right), \lim _{\varepsilon \searrow 0} \nabla u^{\varepsilon}=\nabla u \quad \text { in } L_{\mathrm{loc}}^{2}\left(\mathbb{R}_{+} ; X_{P}\right) \\
\lim _{\varepsilon \searrow 0} \partial_{t} u^{\varepsilon}=\partial_{t} u \text { in } L_{\mathrm{loc}}^{2}\left(\mathbb{R}_{+} ; L^{2}\left(\mathbb{R}^{m}\right)\right), \lim _{\varepsilon \searrow 0} \frac{b \cdot \nabla u^{\varepsilon}}{\varepsilon}=0 \quad \text { in } L_{\mathrm{loc}}^{2}\left(\mathbb{R}_{+} ; L^{2}\left(\mathbb{R}^{m}\right)\right) .
\end{gathered}
$$

Our paper is organized as follows. In Section 2 we discuss the average operators for functions and matrix fields, recalling their main properties. We introduce and study the weighted Sobolev space $H_{P}^{1}$, which will play a crucial role in our analysis. In Section 3 we study the variational solutions of the problems (1), (2) and introduce the limit wave problem which comes out when averaging the matrix field $D$. Section 4 is devoted to the asymptotic analysis. We establish strong and weak convergence results, provided that the initial conditions are well prepared or not. In Section 5 we estimate the propagation speed for the solution of the limit model.

\section{Preliminaries}

We introduce the main functional spaces and tools which allow us to define the average operators along a characteristic flow, for functions and matrix fields. Most of these notions are borrowed from previous works $[4,5,6]$, dealing with the asymptotic analysis of PDEs perturbed by a stiff transport operator. For the completeness of the presentation, we detail them here (or refer to the appendix). We consider the transport operator $\mathcal{T}=\operatorname{div}_{y}(\cdot b)$, defined on

$$
\operatorname{dom} \mathcal{T}=\left\{u \in L^{2}\left(\mathbb{R}^{m}\right): \operatorname{div}_{y}(u b) \in L^{2}\left(\mathbb{R}^{m}\right)\right\} .
$$

The vector field $b$ is assumed to verify the standard hypotheses

$$
b \in W_{\mathrm{loc}}^{1, \infty}\left(\mathbb{R}^{m}\right), \quad \operatorname{div}_{y} b=0
$$


and grows at most linearly at infinity

$$
\exists C>0 \text { such that }|b(y)| \leq C(1+|y|), \quad y \in \mathbb{R}^{m} .
$$

Under the previous hypotheses, the vector field $b$ possesses a global smooth characteristic flow $Y \in W_{\text {loc }}^{1, \infty}\left(\mathbb{R} \times \mathbb{R}^{m}\right)$

$$
\frac{\mathrm{d} Y}{\mathrm{~d} s}=b(Y(s ; y)), \quad(s, y) \in \mathbb{R} \times \mathbb{R}^{m}, \quad Y(0 ; y)=y, \quad y \in \mathbb{R}^{m} .
$$

The vector field $b$ being divergence free, the transformation $y \in \mathbb{R}^{m} \rightarrow Y(s ; y) \in \mathbb{R}^{m}$ is measure preserving for any $s \in \mathbb{R}$. We introduce the $C^{0}$-group of unitary operators $(\zeta(s))_{s \in \mathbb{R}}$ given by

$$
\zeta(s) u=u \circ Y(s ; \cdot), \quad u \in L^{2}\left(\mathbb{R}^{m}\right), \quad s \in \mathbb{R}
$$

whose infinitesimal generator is $\mathcal{T}$. Sometimes we will use the notation $f_{s}(z)=$ $f(Y(s ; z))$, given a function $f=f(y)$.

The orthogonal projection on the kernel of $\mathcal{T}$ coincides with the ergodic mean of the group $(\zeta(s))_{s \in \mathbb{R}}$, thanks to the following classical result in ergodic theory [15].

Theorem 2.1 (von Neumann's ergodic mean theorem)

Let $(\mathcal{G}(s))_{s \in \mathbb{R}}$ be a $C^{0}$-group of unitary operators on a Hilbert space $(\mathcal{H},(\cdot, \cdot))$ and $\mathcal{L}$ be its infinitesimal generator. Then for any $x \in \mathcal{H}$, we have the strong convergence in $\mathcal{H}$

$$
\lim _{S \rightarrow+\infty} \frac{1}{S} \int_{r}^{r+S} \mathcal{G}(s) x \mathrm{~d} s=\operatorname{Proj}_{\operatorname{ker} \mathcal{L}} x, \quad \text { uniformly with respect to } r \in \mathbb{R} .
$$

In particular, the orthogonal projection on $\operatorname{ker} \mathcal{T}=\left\{u \in L^{2}\left(\mathbb{R}^{m}\right): u(Y(s ; \cdot))=\right.$ $u, \forall s \in \mathbb{R}\}$ writes

\section{Proposition 2.1 (Average of $L^{2}\left(\mathbb{R}^{m}\right)$ functions)}

Assume that (3), (4) hold true. Then for any $u \in L^{2}\left(\mathbb{R}^{m}\right)$ we have the strong convergence in $L^{2}\left(\mathbb{R}^{m}\right)$

$$
\lim _{S \rightarrow+\infty} \frac{1}{S} \int_{r}^{r+S} u(Y(s ; \cdot)) \mathrm{d} s=\operatorname{Proj}_{\mathrm{ker}} \mathcal{T} u, \quad \text { uniformly with respect to } r \in \mathbb{R} .
$$

\section{Proof.}

Apply the von Neumann's ergodic mean theorem to the $C^{0}$-group of unitary operators $(\zeta(s))_{s \in \mathbb{R}}$.

In the sequel we use the notation $\langle u\rangle=\lim _{S \rightarrow+\infty} \frac{1}{S} \int_{r}^{r+S} u(Y(s ; \cdot)) \mathrm{d} s, u \in L^{2}\left(\mathbb{R}^{m}\right)$, that is $\langle\cdot\rangle=\operatorname{Proj}_{\text {ker } \mathcal{T}}$. We need to introduce several weighted Lebesgue and Sobolev spaces. For doing that we assume that there is a matrix field $P$ such that

$$
\begin{gathered}
{ }^{t} P=P, \quad P(y) \xi \cdot \xi>0, \quad \xi \in \mathbb{R}^{m} \backslash\{0\}, \quad y \in \mathbb{R}^{m}, \quad P^{-1}, P \in L_{\mathrm{loc}}^{2}\left(\mathbb{R}^{m}\right) \\
{[b, P]:=\left(b \cdot \nabla_{y}\right) P-\partial_{y} b P-P^{t} \partial_{y} b=0, \quad \text { in } \mathcal{D}^{\prime}\left(\mathbb{R}^{m}\right) .}
\end{gathered}
$$

We have the following characterization, in terms of the characteristic flow of $b$, for the matrix fields $A$ satisfying $[b, A]=0$ in $\mathcal{D}^{\prime}\left(\mathbb{R}^{m}\right)$, see Proposition 3.8 [5]. The notation $\partial Y(s ; y)$ stands for the jacobian matrix of the application $y \mapsto Y(s ; y)$. Similarly, the jacobian matrix of the vector field $b$ is denoted by $\partial_{y} b$. 


\section{Proposition 2.2}

Consider $b \in W_{\text {loc }}^{1, \infty}\left(\mathbb{R}^{m}\right)$ (not necessarily divergence free) with at most linear growth at infinity and $A(y) \in L_{\mathrm{loc}}^{1}\left(\mathbb{R}^{m}\right)$. Then $[b, A]:=\left(b \cdot \nabla_{y}\right) A-\partial_{y} b A-A^{t} \partial_{y} b=0$ in $\mathcal{D}^{\prime}\left(\mathbb{R}^{m}\right)$ iff

$$
A(Y(s ; y))=\partial Y(s ; y) A(y)^{t} \partial Y(s ; y), \quad s \in \mathbb{R}, \quad y \in \mathbb{R}^{m} .
$$

Any vector field $c$ in involution with $b$ i.e., $\left(b \cdot \nabla_{y}\right) c-\partial_{y} b c=0$ in $\mathcal{D}^{\prime}\left(\mathbb{R}^{m}\right)$, or equivalently $c(Y(s ; y))=\partial Y(s ; y) c(y), s \in \mathbb{R}, y \in \mathbb{R}^{m}$, provides a symmetric matrix field $P_{c}(y)=$ $c(y) \otimes c(y)$ satisfying $\left[b, P_{c}\right]=0$ in $\mathcal{D}^{\prime}\left(\mathbb{R}^{m}\right)$. Indeed we have

$$
\begin{aligned}
P_{c}(Y(s ; y)) & =c(Y(s ; y)) \otimes c(Y(s ; y)) \\
& =(\partial Y(s ; y) c(y)) \otimes(\partial Y(s ; y) c(y)) \\
& =\partial Y(s ; y)(c(y) \otimes c(y))^{t} \partial Y(s ; y) \\
& =\partial Y(s ; y) P_{c}(y)^{t} \partial Y(s ; y), \quad s \in \mathbb{R}, y \in \mathbb{R}^{m} .
\end{aligned}
$$

If a family $\left\{c_{i}\right\}_{1 \leq i \leq m}$ of vector fields in involution with $b$ is available, and $\left\{c_{i}(y)\right\}_{1 \leq i \leq m}$ form a basis of $\mathbb{R}^{m}$ at any point $y \in \mathbb{R}^{m}$, therefore the symmetric matrix field $P(y)=$ $\sum_{i=1}^{m} c_{i}(y) \otimes c_{i}(y)$ is positive definite and satisfies $[b, P]=0$ in $\mathcal{D}^{\prime}\left(\mathbb{R}^{m}\right)$.

Before going further, let us give an example of a vector field $b$ and matrix field $P$ satisfying (5), (6). We consider $m=2, b(y)=\left(y_{2},-y_{1}\right), y=\left(y_{1}, y_{2}\right) \in \mathbb{R}^{2}$. It is easily seen that $\operatorname{div}_{y} b=0$ and $P=I_{2}$ satisfies (5), (6).

Let us consider the following spaces of matrix fields.

Definition 2.1 We introduce the linear space

$$
H_{Q}=\left\{A: \mathbb{R}^{m} \rightarrow \mathcal{M}_{m}(\mathbb{R}) \text { measurable }: Q^{1 / 2} A Q^{1 / 2} \in L^{2}\right\}
$$

where $Q=P^{-1}$, which is a Hilbert space for the natural scalar product

$$
(A, B)_{H_{Q}}=\int_{\mathbb{R}^{m}} Q^{1 / 2} A Q^{1 / 2}: Q^{1 / 2} B Q^{1 / 2} \mathrm{~d} y=\int_{\mathbb{R}^{m}} Q A: B Q \mathrm{~d} y, \quad \forall A, B \in H_{Q} .
$$

The associated norm is denoted by $|A|_{H_{Q}}$.

Similarly we introduce the Banach space

$$
H_{Q}^{\infty}=\left\{A: \mathbb{R}^{m} \rightarrow \mathcal{M}_{m}(\mathbb{R}) \text { measurable }: Q^{1 / 2} A Q^{1 / 2} \in L^{\infty}\right\},
$$

endowed with the norm

$$
|A|_{H_{Q}^{\infty}}:=\left|Q^{1 / 2} A Q^{1 / 2}\right|_{L^{\infty}}
$$

We also need a localized version of the space $H_{Q}$. For that, we assume that there is a continuous function $\psi$, which is left invariant by the flow of $b$, and goes to infinity when $|y|$ goes to infinity

$$
\psi \in C\left(\mathbb{R}^{m}\right), \quad \psi \circ Y(s ; \cdot)=\psi \text { for any } s \in \mathbb{R}, \lim _{|y| \rightarrow+\infty} \psi(y)=+\infty .
$$

For example, when $m=2, b(y)=\left(y_{2},-y_{1}\right), y=\left(y_{1}, y_{2}\right) \in \mathbb{R}^{2}, P=I_{2}$, we can pick the invariant $\psi(y)=|y|^{2}, y \in \mathbb{R}^{2}$, which satisfies $\lim _{|y| \rightarrow+\infty} \psi(y)=+\infty$.

We consider the local space

$$
H_{Q, \text { loc }}=\left\{A: \mathbb{R}^{m} \rightarrow \mathcal{M}_{m}(\mathbb{R}) \text { measurable }: \mathbf{1}_{\{\psi \leq k\}} A \in H_{Q} \text { for any } k \in \mathbb{N}\right\}
$$


We say that a family $\left(A_{i}\right)_{i} \subset H_{Q \text {,loc }}$ converges in $H_{Q \text {,loc }}$ toward some $A \in H_{Q \text {,loc }}$ iff for any $k \in \mathbb{N}$, the family $\left(\mathbf{1}_{\{\psi \leq k\}} A_{i}\right)_{i}$ converges in $H_{Q}$ toward $\mathbf{1}_{\{\psi \leq k\}} A$. The space $H_{Q \text {,loc }}$, as well as the convergence in this space, are not depending on the choice of the function $\psi$ satisfying (7). Notice that we have the continuous inclusion $H_{Q} \subset H_{Q \text {,loc }}$. We introduce the family of linear transformations $(G(s))_{s \in \mathbb{R}}$, acting on $H_{Q}$ and also on $H_{Q \text {,loc }}$. For any matrix field $A$ we use the notation $A_{s}=A \circ Y(s ; \cdot)$. The following result is borrowed from [3], see Proposition 4.1. The proof details are postponed to appendix A.

\section{Proposition 2.3}

Assume that the hypotheses (3), (4), (5), (6) hold true and that $Q=P^{-1}$.

1. The family of applications

$$
A \mapsto G(s) A:=\partial Y^{-1}(s ; \cdot) A_{s}{ }^{t} \partial Y^{-1}(s ; \cdot)=\partial Y(-s ; Y(s ; \cdot)) A_{s}{ }^{t} \partial Y(-s ; Y(s ; \cdot))
$$

is a $C^{0}$-group of unitary operators on $H_{Q}$.

2. If $A$ is a field of symmetric matrices, then so is $G(s) A$, for any $s \in \mathbb{R}$.

3. If $A$ is a field of positive semi-definite matrices, then so is $G(s) A$, for any $s \in \mathbb{R}$.

4. Let $\mathcal{S} \subset \mathbb{R}^{m}$ be an invariant set of the flow of b, that is $Y(s ; \mathcal{S})=\mathcal{S}$, for any $s \in \mathbb{R}$. If there is $d>0$ such that $Q^{1 / 2}(y) A(y) Q^{1 / 2}(y) \geq d I_{m}, y \in \mathcal{S}$, then for any $s \in \mathbb{R}$ we have $Q^{1 / 2}(y)(G(s) A)(y) Q^{1 / 2}(y) \geq d I_{m}, y \in \mathcal{S}$.

5. Moreover, if (7) holds true, then the family of applications $(G(s))_{s \in \mathbb{R}}$ acts on $H_{Q \text {,loc }}$, that is, if $A \in H_{Q \text {,loc }}$, then $G(s) A \in H_{Q, \text { loc }}$ for any $s \in \mathbb{R}$. We have

$$
\mathbf{1}_{\{\psi \leq k\}} G(s) A=G(s)\left(\mathbf{1}_{\{\psi \leq k\}} A\right), \quad A \in H_{Q, \text { loc }}, \quad s \in \mathbb{R}, \quad k \in \mathbb{N} .
$$

The infinitesimal generator of the group $G$ is given by

$$
L: \operatorname{dom} L \subset H_{Q} \rightarrow H_{Q}, \operatorname{dom} L=\left\{A \in H_{Q}: \exists \lim _{s \rightarrow 0} \frac{G(s) A-A}{s} \text { in } H_{Q}\right\}
$$

and $L A=\lim _{s \rightarrow 0} \frac{G(s) A-A}{s}$ for any $A \in \operatorname{dom} L$. Notice that $C_{c}^{1}\left(\mathbb{R}^{m}\right) \subset \operatorname{dom} L$ and $L A=\left(b \cdot \nabla_{y}\right) A-\partial_{y} b \stackrel{s}{A}-A^{t} \partial_{y} b, A \in C_{c}^{1}\left(\mathbb{R}^{m}\right)$ (use the hypothesis $Q \in L_{\text {loc }}^{2}\left(\mathbb{R}^{m}\right)$ and the dominated convergence theorem). For the main properties of the operator $L$ we refer to [5], Proposition 3.13 .

\section{Proposition 2.4}

Assume that the hypotheses (3), (4), (5), (6) hold true.

1. The domain of $L$ is dense in $H_{Q}$ and $L$ is closed.

2. The matrix field $A \in H_{Q}$ belongs to $\operatorname{dom} L$ iff there is a constant $C>0$ such that

$$
|G(s) A-A|_{H_{Q}} \leq C|s|, \quad s \in \mathbb{R} .
$$

3. The operator $L$ is skew-adjoint and we have the orthogonal decomposition $H_{Q}=$ ker $L \stackrel{\perp}{\oplus} \overline{\text { Range } L \text {. }}$ 
The transformations $(G(s))_{s \in \mathbb{R}}$ also operate on $H_{Q}^{\infty}$. More exactly, for any $s \in \mathbb{R}$, and any $A \in H_{Q}^{\infty}$, we have $G(s) A \in H_{Q}^{\infty}$ and $|G(s) A|_{H_{Q}^{\infty}}=|A|_{H_{Q}^{\infty}}$. Indeed, thanks to (19) and to the orthogonality of $\mathcal{O}(s ; \cdot)$ (see the proof of Proposition 2.3 for the definition of the matrix field $\mathcal{O})$, observe that

$$
\begin{aligned}
Q^{1 / 2} G(s) A Q^{1 / 2}: Q^{1 / 2} G(s) A Q^{1 / 2} & ={ }^{t} \mathcal{O}(s ; \cdot) Q_{s}^{1 / 2} A_{s} Q_{s}^{1 / 2} \mathcal{O}(s ; \cdot):{ }^{t} \mathcal{O}(s ; \cdot) Q_{s}^{1 / 2} A_{s} Q_{s}^{1 / 2} \mathcal{O}(s ; \cdot) \\
& =\left(Q^{1 / 2} A Q^{1 / 2}: Q^{1 / 2} A Q^{1 / 2}\right)_{s}, \quad s \in \mathbb{R}
\end{aligned}
$$

and our claim follows immediately. Applying Theorem 2.1 to the group $(G(s))_{s \in \mathbb{R}}$, we deduce that the average of a matrix field $\langle A\rangle:=\lim _{S \rightarrow+\infty} \frac{1}{S} \int_{r}^{r+S} G(s) A$ ds is well defined and coincides with the orthogonal projection on $\operatorname{ker} L$. Moreover, by Proposition 2.3, $(G(s))_{s \in \mathbb{R}}$ also acts on $H_{Q \text {,loc }}$, and any matrix field of $H_{Q}^{\infty} \subset H_{Q \text {,loc }}$ possesses an average in $H_{Q \text {,loc }}$, still denoted by $\langle\cdot\rangle$ as for the matrix fields in $H_{Q}$, cf. Theorem $3.2[2]$ (see Appendix A for proof details).

\section{Theorem 2.2 (Average of $H_{Q \text {,loc }}$ matrix fields)}

Assume that (3), (4), (5), (6) hold true and that $Q=P^{-1}$.

1. For any matrix field $A \in H_{Q}$ we have the strong convergence in $H_{Q}$

$$
\langle A\rangle:=\lim _{S \rightarrow+\infty} \frac{1}{S} \int_{r}^{r+S} \partial Y(-s ; Y(s ; \cdot)) A(Y(s ; \cdot))^{t} \partial Y(-s ; Y(s ; \cdot)) \mathrm{d} s=\operatorname{Proj}_{\operatorname{ker} L} A
$$

uniformly with respect to $r \in \mathbb{R}$.

2. If $A \in H_{Q}$ is a field of symmetric positive semi-definite matrices, then so is $\langle A\rangle$.

3. Let $\mathcal{S} \subset \mathbb{R}^{m}$ be an invariant set of the flow of b, that is $Y(s ; \mathcal{S})=\mathcal{S}$ for any $s \in \mathbb{R}$. If $A \in H_{Q}$ and there is $d>0$ such that

$$
Q^{1 / 2}(y) A(y) Q^{1 / 2}(y) \geq d I_{m}, \quad y \in \mathcal{S}
$$

therefore we have

$$
Q^{1 / 2}(y)\langle A\rangle(y) Q^{1 / 2}(y) \geq d I_{m}, \quad y \in \mathcal{S}
$$

and in particular, $\langle A\rangle(y)$ is definite positive, $y \in \mathcal{S}$.

4. If $A \in H_{Q} \cap H_{Q}^{\infty}$, then $\langle A\rangle \in H_{Q} \cap H_{Q}^{\infty}$ and

$$
|\langle A\rangle|_{H_{Q}} \leq|A|_{H_{Q}}, \quad|\langle A\rangle|_{H_{Q}^{\infty}} \leq|A|_{H_{Q}^{\infty}}
$$

5. Moreover, assume that (7) holds true. For any matrix field $A \in H_{Q \text {,loc }}$, the family

$$
\left(\frac{1}{S} \int_{r}^{r+S} \partial Y(-s ; Y(s ; \cdot)) A(Y(s ; \cdot))^{t} \partial Y(-s ; Y(s ; \cdot)) \mathrm{d} s\right)_{S>0}
$$

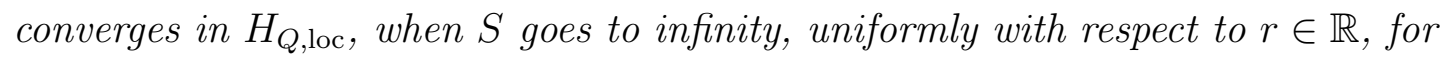
any fixed $k \in \mathbb{N}$. Its limit, denoted by $\langle A\rangle$, satisfies

$$
\mathbf{1}_{\{\psi \leq k\}}\langle A\rangle=\left\langle\mathbf{1}_{\{\psi \leq k\}} A\right\rangle, \text { for any } k \in \mathbb{N}
$$


where the symbol $\langle\cdot\rangle$ in the right hand side stands for the average operator on $H_{Q}$. In particular, any matrix field $A \in H_{Q}^{\infty}$ has an average in $H_{Q, \text { loc }}$ and $|\langle A\rangle|_{H_{Q}^{\infty}} \leq$ $|A|_{H_{Q}^{\infty}}$. If $A \in H_{Q, \text { loc }}$ is such that

$$
Q^{1 / 2}(y) A(y) Q^{1 / 2}(y) \geq \alpha I_{m}, \quad y \in \mathbb{R}^{m},
$$

for some $\alpha>0$, then we have

$$
Q^{1 / 2}(y)\langle A\rangle(y) Q^{1 / 2}(y) \geq \alpha I_{m}, \quad y \in \mathbb{R}^{m} .
$$

We also introduce the linear spaces of vector fields

$$
\begin{gathered}
X_{Q}=\left\{c: \mathbb{R}^{m} \rightarrow \mathbb{R}^{m} \text { measurable }: \int_{\mathbb{R}^{m}} Q(y): c(y) \otimes c(y) \mathrm{d} y<+\infty\right\} \\
X_{Q}^{\infty}=\left\{c: \mathbb{R}^{m} \rightarrow \mathbb{R}^{m} \text { measurable }:\left|Q^{1 / 2} c\right| \in L^{\infty}\left(\mathbb{R}^{m}\right)\right\} .
\end{gathered}
$$

The linear space $X_{Q}$, endowed with the scalar product

$$
(\cdot, \cdot)_{X_{Q}}: X_{Q} \times X_{Q} \rightarrow \mathbb{R}, \quad(c, d)_{X_{Q}}=\int_{\mathbb{R}^{m}} Q(y): c(y) \otimes d(y) \mathrm{d} y, \quad c, d \in X_{Q}
$$

becomes a Hilbert space, whose norm is denoted by $|c|_{X_{Q}}=(c, c)_{X_{Q}}^{1 / 2}, c \in X_{Q}$.

The linear space $X_{Q}^{\infty}$ is a Banach space with respect to the norm

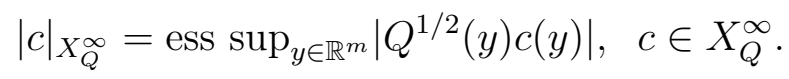

Notice that for any $c \in X_{Q} \cap X_{Q}^{\infty}$, we have $c \otimes c \in H_{Q} \cap H_{Q}^{\infty}$ and

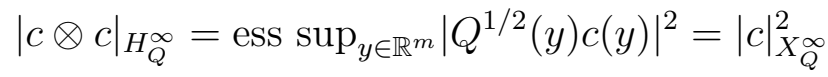

$$
\begin{aligned}
& |c \otimes c|_{H_{Q}}=\left(\int_{\mathbb{R}^{m}}\left|Q^{1 / 2}(y) c(y)\right|^{4} \mathrm{~d} y\right)^{1 / 2} \leq|c|_{X_{Q}}|c|_{X_{Q}^{\infty}} .
\end{aligned}
$$

Replacing the matrix field $Q$ by the matrix field $P$, we obtain the linear spaces $X_{P}, X_{P}^{\infty}$. When applying variational methods, we need a weighted $H^{1}$ space. We consider the linear subspace made of $L^{2}\left(\mathbb{R}^{m}\right)$ functions, whose gradient belongs to $X_{P}$

$$
H_{P}^{1}=\left\{u \in L^{2}\left(\mathbb{R}^{m}\right): \nabla_{y} u \in X_{P}\right\}
$$

which is a Hilbert space, with the scalar product

$$
(u, v)_{H_{P}^{1}}=\int_{\mathbb{R}^{m}} u(y) v(y) \mathrm{d} y+\int_{\mathbb{R}^{m}} P(y): \nabla_{y} u \otimes \nabla_{y} v \mathrm{~d} y, \quad u, v \in H_{P}^{1} .
$$

The $C^{0}$-group $(\zeta(s))_{s \in \mathbb{R}}$ acts on $H_{P}^{1}$ cf. Proposition 3.5 [2] (see Appendix A for proof details). 


\section{Proposition 2.5 (Average of $H_{P}^{1}$ functions)}

Assume that the hypotheses (3), (4), (5), (6) hold true. For any $s \in \mathbb{R}$ and $u \in H_{P}^{1}$ we have $u_{s} \in H_{P}^{1}$ and $\left|u_{s}\right|_{H_{P}^{1}}=|u|_{H_{P}^{1}}$. The family of applications $u \in H_{P}^{1} \rightarrow \zeta^{1}(s) u=$ $u \circ Y(s ; \cdot) \in H_{P}^{1}$ is a $C^{0}$-group of unitary operators on $H_{P}^{1}$. In particular, for any $u \in H_{P}^{1}$ we have $\langle u\rangle \in H_{P}^{1}$

$$
\begin{gathered}
\nabla_{y}\langle u\rangle=\lim _{S \rightarrow+\infty} \frac{1}{S} \int_{r}^{r+S} \nabla_{y} u_{s} \mathrm{~d} s, \quad \text { strongly in } X_{P}, \text { uniformly with respect to } r \in \mathbb{R} \\
u-\langle u\rangle \perp \operatorname{ker} \mathcal{T} \cap H_{P}^{1} \text { in } H_{P}^{1}, \quad\left|\nabla_{y}\langle u\rangle\right|_{X_{P}} \leq\left|\nabla_{y} u\right|_{X_{P}} .
\end{gathered}
$$

For further developments, we introduce the following result, borrowed from [2], Lemma 5.1 (see Appendix A for proof details).

\section{Lemma 2.1}

Assume that the hypotheses (3), (4), (5), (6), (7) hold true. For any matrix field $D \in H_{Q}^{\infty}$ and any vector field $c \in X_{P}$ we have the convergence

$$
\lim _{S \rightarrow+\infty} \frac{1}{S} \int_{r}^{r+S} G(s) D c \mathrm{~d} s=\langle D\rangle c, \text { strongly in } X_{Q} \text {, uniformly with respect to } r \in \mathbb{R} \text {. }
$$

\section{Variational solutions}

We appeal to variational methods for solving (1), (2). Under the hypotheses (3), (4), (5), (6), we consider the continuous embedding of separable Hilbert spaces $H_{P}^{1} \hookrightarrow$ $L^{2}\left(\mathbb{R}^{m}\right)$, with dense image (since $C_{c}^{1}\left(\mathbb{R}^{m}\right) \subset H_{P}^{1}$ ) and the bilinear forms $a^{\varepsilon}: H_{P}^{1} \times H_{P}^{1} \rightarrow$ $\mathbb{R},\langle a\rangle: H_{P}^{1} \times H_{P}^{1} \rightarrow \mathbb{R}$ given by

$$
\begin{gathered}
a^{\varepsilon}(u, v)=\int_{\mathbb{R}^{m}} D(y) \nabla u \cdot \nabla v \mathrm{~d} y+\frac{1}{\varepsilon^{2}} \int_{\mathbb{R}^{m}}(b \cdot \nabla u)(b \cdot \nabla v) \mathrm{d} y, \quad u, v \in H_{P}^{1} \\
\langle a\rangle(u, v)=\int_{\mathbb{R}^{m}}\langle D\rangle(y) \nabla u \cdot \nabla v \mathrm{~d} y, \quad u, v \in H_{P}^{1} .
\end{gathered}
$$

We assume that $D=D(y)$ is a field of symmetric non-negative matrices, satisfying

$$
Q^{1 / 2}(y)(D(y)+b(y) \otimes b(y)) Q^{1 / 2}(y) \geq d I_{m}, \quad y \in \mathbb{R}^{m}
$$

for some constant $d>0$. We suppose also that

$$
D \in H_{Q}^{\infty}, \quad b \in X_{Q}^{\infty} .
$$

\section{Proposition 3.1}

Assume that the hypotheses (3), (4), (5), (6), (9), (10) hold true. The bilinear forms $a^{\varepsilon}$ are well defined, continuous, symmetric, non-negative. For $\left.\left.\varepsilon \in\right] 0,1\right]$, the forms $a^{\varepsilon}$ are coercive on $H_{P}^{1}$, with respect to $L^{2}\left(\mathbb{R}^{m}\right)$.

Proof. For any $u, v \in H_{P}^{1}$ we have

$$
|D \nabla u \cdot \nabla v|=\left|Q^{1 / 2} D Q^{1 / 2}:\left(P^{1 / 2} \nabla v\right) \otimes\left(P^{1 / 2} \nabla u\right)\right| \leq|D|_{H_{Q}^{\infty}}\left|P^{1 / 2} \nabla v\right|\left|P^{1 / 2} \nabla u\right|
$$


and

$$
\begin{aligned}
|(b \cdot \nabla u)(b \cdot \nabla v)| & =\left|Q^{1 / 2} b \otimes b Q^{1 / 2}:\left(P^{1 / 2} \nabla v\right) \otimes\left(P^{1 / 2} \nabla u\right)\right| \\
& \leq|b \otimes b|_{H_{Q}^{\infty}}\left|P^{1 / 2} \nabla v\right|\left|P^{1 / 2} \nabla u\right|=|b|_{X_{Q}^{\infty}}^{2}\left|P^{1 / 2} \nabla v\right|\left|P^{1 / 2} \nabla u\right| .
\end{aligned}
$$

We deduce that

$$
\left|a^{\varepsilon}(u, v)\right| \leq\left(|D|_{H_{Q}^{\infty}}+\frac{|b|_{X_{Q}^{\infty}}^{2}}{\varepsilon^{2}}\right)|u|_{H_{P}^{1}}|v|_{H_{P}^{1}}
$$

saying that $a^{\varepsilon}$ is well defined, and continuous on $H_{P}^{1}$. It is also symmetric and nonnegative, thanks to the symmetry and non-negativity of $D(y), y \in \mathbb{R}^{m}$. The coercivity comes by (9), observing that for any $u \in H_{P}^{1}, 0<\varepsilon \leq 1$ we have

$$
\begin{aligned}
a^{\varepsilon}(u, u)+d|u|_{L^{2}\left(\mathbb{R}^{m}\right)}^{2} & =\int_{\mathbb{R}^{m}} Q^{1 / 2}\left(D+\frac{b \otimes b}{\varepsilon^{2}}\right) Q^{1 / 2}:\left(P^{1 / 2} \nabla u\right) \otimes\left(P^{1 / 2} \nabla u\right) \mathrm{d} y+d|u|_{L^{2}\left(\mathbb{R}^{m}\right)}^{2} \\
& \geq d|\nabla u|_{X_{P}}^{2}+d|u|_{L^{2}\left(\mathbb{R}^{m}\right)}^{2}=d|u|_{H_{P}^{1}}^{2} .
\end{aligned}
$$

\section{Remark 3.1}

For any $u \in H_{P}^{1}$ we have

$$
|b \cdot \nabla u|_{L^{2}\left(\mathbb{R}^{m}\right)}^{2}=\int_{\mathbb{R}^{m}}\left(Q^{1 / 2} b \cdot P^{1 / 2} \nabla u\right)^{2} \mathrm{~d} y \leq|b|_{X_{Q}^{\infty}}^{2}|\nabla u|_{X_{P}}^{2} .
$$

Since for any test function $\varphi \in C_{c}^{1}\left(\mathbb{R}^{m}\right)$ we have

$$
\int_{\mathbb{R}^{m}} u b \cdot \nabla \varphi \mathrm{d} y=\int_{\mathbb{R}^{m}} u \operatorname{div}_{y}(\varphi b) \mathrm{d} y=-\int_{\mathbb{R}^{m}} \nabla u \cdot b \varphi(y) \mathrm{d} y
$$

we deduce that $u \in \operatorname{dom} \mathcal{T}, \mathcal{T} u=b \cdot \nabla u$. Therefore we proved that the inclusion $H_{P}^{1} \subset \operatorname{dom} \mathcal{T}$ is continuous

$$
|u|_{L^{2}\left(\mathbb{R}^{m}\right)}^{2}+|\mathcal{T} u|_{L^{2}\left(\mathbb{R}^{m}\right)}^{2} \leq|u|_{L^{2}\left(\mathbb{R}^{m}\right)}^{2}+|b|_{X_{Q}^{\infty}}^{2}|\nabla u|_{X_{P}}^{2} \leq \max \left\{1,|b|_{X_{Q}^{\infty}}^{2}\right\}|u|_{H_{P}^{1}}^{2}, \quad u \in H_{P}^{1} .
$$

By standard results we obtain the following proposition.

\section{Proposition 3.2}

Assume that the hypotheses of Proposition 3.1 hold true. Let $\left(u_{\mathrm{in}}^{\varepsilon}, \dot{u}_{\mathrm{in}}^{\varepsilon}\right) \in H_{P}^{1} \times L^{2}\left(\mathbb{R}^{m}\right)$. For any $\varepsilon \in] 0,1]$ there is a unique variational solution of $(1),(2)$ i.e., $u^{\varepsilon} \in L_{\text {loc }}^{\infty}\left(\mathbb{R}_{+} ; H_{P}^{1}\right)$, $\partial_{t} u^{\varepsilon} \in L_{\mathrm{loc}}^{\infty}\left(\mathbb{R}_{+} ; L^{2}\left(\mathbb{R}^{m}\right)\right)$

$\left(u^{\varepsilon}(0), \partial_{t} u^{\varepsilon}(0)\right)=\left(u_{\mathrm{in}}^{\varepsilon}, \dot{u}_{\mathrm{in}}^{\varepsilon}\right), \frac{\mathrm{d}}{\mathrm{d} t} \int_{\mathbb{R}^{m}} \partial_{t} u^{\varepsilon} v(y) \mathrm{d} y+a^{\varepsilon}\left(u^{\varepsilon}(t), v\right)=0 \quad$ in $\mathcal{D}^{\prime}\left(\mathbb{R}_{+}\right), v \in H_{P}^{1}$.

Moreover we have $u^{\varepsilon} \in C\left(\mathbb{R}_{+} ; H_{P}^{1}\right), \partial_{t} u^{\varepsilon} \in C\left(\mathbb{R}_{+} ; L^{2}\left(\mathbb{R}^{m}\right)\right)$ and for any $t \in \mathbb{R}_{+}, \quad 0<$ $\varepsilon \leq 1$

$$
\begin{aligned}
\left|\partial_{t} u^{\varepsilon}(t)\right|_{L^{2}\left(\mathbb{R}^{m}\right)}^{2} & +d\left|\nabla u^{\varepsilon}(t)\right|_{X_{P}}^{2}+\left(\frac{1}{\varepsilon^{2}}-1\right)\left|b \cdot \nabla u^{\varepsilon}(t)\right|_{L^{2}\left(\mathbb{R}^{m}\right)}^{2} \leq\left|\dot{u}_{\mathrm{in}}^{\varepsilon}\right|_{L^{2}\left(\mathbb{R}^{m}\right)}^{2}+|D|_{H_{Q}^{\infty}}\left|\nabla u_{\mathrm{in}}^{\varepsilon}\right|_{X_{P}}^{2} \\
& +\frac{1}{\varepsilon^{2}}\left|b \cdot \nabla u_{\mathrm{in}}^{\varepsilon}\right|_{L^{2}\left(\mathbb{R}^{m}\right)}^{2}
\end{aligned}
$$

and

$$
\left|u^{\varepsilon}(t)\right|_{L^{2}\left(\mathbb{R}^{m}\right)}^{2} \leq 2\left|u_{\text {in }}^{\varepsilon}\right|_{L^{2}\left(\mathbb{R}^{m}\right)}^{2}+2 t^{2}\left[\left|\dot{u}_{\text {in }}^{\varepsilon}\right|_{L^{2}\left(\mathbb{R}^{m}\right)}^{2}+|D|_{H_{Q}^{\infty}}\left|\nabla u_{\text {in }}^{\varepsilon}\right|_{X_{P}}^{2}+\frac{1}{\varepsilon^{2}}\left|b \cdot \nabla u_{\text {in }}^{\varepsilon}\right|_{L^{2}\left(\mathbb{R}^{m}\right)}^{2}\right] .
$$


Proof. This is a direct consequence of [8] chapter XVIII, section 5, see also [14]. By Proposition 3.1 we know that, for any $\varepsilon \in] 0,1]$, the bilinear form $a^{\varepsilon}$ is coercive on $H_{P}^{1}$ with respect to $L^{2}\left(\mathbb{R}^{m}\right)$. We deduce that, for any $\left(u_{\text {in }}^{\varepsilon}, \dot{u}_{\text {in }}^{\varepsilon}\right) \in H_{P}^{1} \times L^{2}\left(\mathbb{R}^{m}\right)$, there is a unique variational solution $u^{\varepsilon}$ for (1), (2). By the energy balance we obtain for any $\left.\left.t \in \mathbb{R}_{+}, \varepsilon \in\right] 0,1\right]$

$$
\left|\partial_{t} u^{\varepsilon}(t)\right|_{L^{2}\left(\mathbb{R}^{m}\right)}^{2}+a^{\varepsilon}\left(u^{\varepsilon}(t), u^{\varepsilon}(t)\right)=\left|\dot{u}_{\mathrm{in}}^{\varepsilon}\right|_{L^{2}\left(\mathbb{R}^{m}\right)}^{2}+a^{\varepsilon}\left(u_{\mathrm{in}}^{\varepsilon}, u_{\mathrm{in}}^{\varepsilon}\right)
$$

implying that

$$
\begin{aligned}
\left|\partial_{t} u^{\varepsilon}(t)\right|_{L^{2}\left(\mathbb{R}^{m}\right)}^{2} & +d\left|\nabla u^{\varepsilon}(t)\right|_{X_{P}}^{2}+\left(\frac{1}{\varepsilon^{2}}-1\right)\left|b \cdot \nabla u^{\varepsilon}(t)\right|_{L^{2}\left(\mathbb{R}^{m}\right)}^{2} \\
& \leq\left|\dot{u}_{\text {in }}^{\varepsilon}\right|_{L^{2}\left(\mathbb{R}^{m}\right)}^{2}+|D|_{H_{Q}^{\infty}}\left|\nabla u_{\text {in }}^{\varepsilon}\right|_{X_{P}}^{2}+\frac{1}{\varepsilon^{2}}\left|b \cdot \nabla u_{\text {in }}^{\varepsilon}\right|_{L^{2}\left(\mathbb{R}^{m}\right)}^{2}, t \in \mathbb{R}_{+} .
\end{aligned}
$$

As we know that $\partial_{t} u^{\varepsilon} \in C\left(\mathbb{R}_{+} ; L^{2}\left(\mathbb{R}^{m}\right)\right)$, we can write

$$
u^{\varepsilon}(t)=u^{\varepsilon}(0)+\int_{0}^{t} \partial_{t} u^{\varepsilon}(s) \mathrm{d} s, \quad t \in \mathbb{R}_{+}
$$

implying that for any $t \in \mathbb{R}_{+}$

$$
\begin{aligned}
\left|u^{\varepsilon}(t)\right|_{L^{2}\left(\mathbb{R}^{m}\right)}^{2} & \leq\left(\left|u_{\mathrm{in}}^{\varepsilon}\right|_{L^{2}\left(\mathbb{R}^{m}\right)}+\int_{0}^{t}\left|\partial_{t} u^{\varepsilon}(s)\right|_{L^{2}\left(\mathbb{R}^{m}\right)} \mathrm{d} s\right)^{2} \\
& \leq 2\left|u_{\mathrm{in}}^{\varepsilon}\right|_{L^{2}\left(\mathbb{R}^{m}\right)}^{2}+2 t \int_{0}^{t}\left|\partial_{t} u^{\varepsilon}(s)\right|_{L^{2}\left(\mathbb{R}^{m}\right)}^{2} \mathrm{~d} s \\
& \leq 2\left|u_{\mathrm{in}}^{\varepsilon}\right|_{L^{2}\left(\mathbb{R}^{m}\right)}^{2}+2 t^{2}\left[\left|\dot{u}_{\mathrm{in}}^{\varepsilon}\right|_{L^{2}\left(\mathbb{R}^{m}\right)}^{2}+|D|_{H_{Q}^{\infty}}\left|\nabla u_{\mathrm{in}}^{\varepsilon}\right|_{X_{P}}^{2}+\frac{1}{\varepsilon^{2}}\left|b \cdot \nabla u_{\mathrm{in}}^{\varepsilon}\right|_{L^{2}\left(\mathbb{R}^{m}\right)}^{2}\right] .
\end{aligned}
$$

The bilinear form $\langle a\rangle$ will enter the variational formulation of the limit model, and corresponds to a wave operator as well. As the average matrix field $\langle D\rangle$ inherits the properties of the matrix field $D$, we prove the following result.

\section{Proposition 3.3}

Assume that the hypotheses (3), (4), (5), (6), (7), (9), (10) hold true. The bilinear form $\langle a\rangle$ is well defined, continuous, symmetric, non-negative and coercive on $H_{P}^{1}$, with respect to $\operatorname{dom} \mathcal{T} \subset L^{2}\left(\mathbb{R}^{m}\right)$.

Proof. The embedding $H_{P}^{1} \hookrightarrow \operatorname{dom} \mathcal{T}$ is continuous cf. Remark 3.1 and has dense image (since $C_{c}^{1}\left(\mathbb{R}^{m}\right)$ is dense in $\operatorname{dom} \mathcal{T}$ ). For any $u, v \in H_{P}^{1}$ we have

$$
\int_{\mathbb{R}^{m}}|\langle D\rangle(y) \nabla u \cdot \nabla v| \mathrm{d} y \leq|\langle D\rangle|_{H_{Q}^{\infty}}|\nabla u|_{X_{P}}|\nabla v|_{X_{P}} \leq|D|_{H_{Q}^{\infty}}|u|_{H_{P}^{1}}|v|_{H_{P}^{1}}
$$

saying that $\langle a\rangle$ is continuous. By Theorem 2.2 we know that $\langle D\rangle$ is symmetric and non-negative, implying that $\langle a\rangle$ is symmetric and non-negative. The coercivity of $\langle a\rangle$ 
comes easily, thanks to the last statement in Theorem 2.2, by noticing that for any $u \in H_{P}^{1}$ we can write

$$
\begin{aligned}
\langle a\rangle(u, u)+d|u|_{L^{2}\left(\mathbb{R}^{m}\right)}^{2} & +|\mathcal{T} u|_{L^{2}\left(\mathbb{R}^{m}\right)}^{2}=\int_{\mathbb{R}^{m}}\langle D\rangle \nabla u \cdot \nabla u \mathrm{~d} y+d|u|_{L^{2}\left(\mathbb{R}^{m}\right)}^{2}+|\mathcal{T} u|_{L^{2}\left(\mathbb{R}^{m}\right)}^{2} \\
& =\int_{\mathbb{R}^{m}}(\langle D\rangle+b \otimes b) \nabla u \cdot \nabla u \mathrm{~d} y+d|u|_{L^{2}\left(\mathbb{R}^{m}\right)}^{2} \\
& =\int_{\mathbb{R}^{m}} Q^{1 / 2}(\langle D\rangle+b \otimes b) Q^{1 / 2}: P^{1 / 2} \nabla u \otimes P^{1 / 2} \nabla u \mathrm{~d} y+d|u|_{L^{2}\left(\mathbb{R}^{m}\right)}^{2} \\
& \geq d|\nabla u|_{X_{P}}^{2}+d|u|_{L^{2}\left(\mathbb{R}^{m}\right)}^{2}=d|u|_{H_{P}^{1}}^{2} .
\end{aligned}
$$

We inquire now about the well posedness of the problem

$$
\begin{gathered}
\partial_{t}^{2} u-\operatorname{div}_{y}(\langle D\rangle(y) \nabla u)=0, \quad(t, y) \in \mathbb{R}_{+} \times \mathbb{R}^{m} \\
u(0, y)=u_{\text {in }}(y), \quad \partial_{t} u(0, y)=\dot{u}_{\text {in }}(y), \quad y \in \mathbb{R}^{m} .
\end{gathered}
$$

\section{Proposition 3.4}

Assume that the hypotheses of Proposition 3.3 hold true. For any $\left(u_{\mathrm{in}}, \dot{u}_{\mathrm{in}}\right) \in H_{P}^{1} \times$ $\operatorname{dom} \mathcal{T}$ there is a unique variational solution of (11), (12) i.e., $u \in L_{\mathrm{loc}}^{\infty}\left(\mathbb{R}_{+} ; H_{P}^{1}\right), \partial_{t} u \in$ $L_{\text {loc }}^{\infty}\left(\mathbb{R}_{+} ; \operatorname{dom} \mathcal{T}\right)$

$$
\left(u(0)=u_{\text {in }}, \partial_{t} u(0)=\dot{u}_{\text {in }}\right), \quad \frac{\mathrm{d}}{\mathrm{d} t} \int_{\mathbb{R}^{m}} \partial_{t} u v(y) \mathrm{d} y+\langle a\rangle(u(t), v)=0 \quad \text { in } \mathcal{D}^{\prime}\left(\mathbb{R}_{+}\right), v \in H_{P}^{1} .
$$

Moreover we have $u \in C\left(\mathbb{R}_{+} ; H_{P}^{1}\right), \partial_{t} u \in C\left(\mathbb{R}_{+} ; \operatorname{dom} \mathcal{T}\right)$ and for any $t \in \mathbb{R}_{+}$

$$
\begin{gathered}
\left|\partial_{t} u(t)\right|_{\text {dom } \mathcal{T}}^{2} \leq\left|\dot{u}_{\text {in }}\right|_{\text {dom } \mathcal{T}}^{2}+|D|_{H_{Q}^{\infty}}\left|\nabla u_{\text {in }}\right|_{X_{P}}^{2} \\
d|u(t)|_{H_{P}^{1}}^{2} \leq \max \{1, d\}\left[2 \max \left\{1,|b|_{X_{Q}^{\infty}}^{2}\right\}\left|u_{\text {in }}\right|_{H_{P}^{1}}^{2}+2 t^{2}\left(\left|\dot{u}_{\text {in }}\right|_{\text {dom } \mathcal{T}}^{2}+|D|_{H_{Q}^{\infty}}\left|\nabla u_{\text {in }}\right|_{X_{P}}^{2}\right)\right] \\
+\left|\dot{u}_{\text {in }}\right|_{\operatorname{dom} \mathcal{T}}^{2}+|D|_{H_{Q}^{\infty}}\left|\nabla u_{\text {in }}\right|_{X_{P}}^{2} .
\end{gathered}
$$

If $\left(u_{\text {in }}, \dot{u}_{\text {in }}\right) \in\left(H_{P}^{1} \cap \operatorname{ker} \mathcal{T}\right) \times \operatorname{ker} \mathcal{T}$, then $\left(u(t), \partial_{t} u(t)\right) \in\left(H_{P}^{1} \cap \operatorname{ker} \mathcal{T}\right) \times \operatorname{ker} \mathcal{T}$ for any $t \in \mathbb{R}_{+}$.

Proof. It is enough to apply the standard results $[14,8]$ with the bilinear form $\langle a\rangle: H_{P}^{1} \times H_{P}^{1} \rightarrow \mathbb{R}$ and the embedding $H_{P}^{1} \subset \operatorname{dom} \mathcal{T}$. By the energy conservation we have

$$
\begin{aligned}
\left|\partial_{t} u(t)\right|_{\text {dom } \mathcal{T}}^{2} & \leq\left|\partial_{t} u(t)\right|_{\text {dom } \mathcal{T}}^{2}+\langle a\rangle(u(t), u(t)) \\
& =\left|\dot{u}_{\text {in }}\right|_{\text {dom } \mathcal{T}}^{2}+\langle a\rangle\left(u_{\text {in }}, u_{\text {in }}\right) \\
& \leq\left|\dot{u}_{\text {in }}\right|_{\text {dom } \mathcal{T}}^{2}+|D|_{H_{Q}^{\infty}}\left|\nabla u_{\text {in }}\right|_{X_{P}}^{2}
\end{aligned}
$$


But we can write, cf. Theorem 2.2

$$
\begin{aligned}
d|\nabla u(t)|_{X_{P}}^{2} & \leq \int_{\mathbb{R}^{m}}(\langle D\rangle+b \otimes b) \nabla u(t) \cdot \nabla u(t) \mathrm{d} y \\
& =|\mathcal{T} u(t)|_{L^{2}\left(\mathbb{R}^{m}\right)}^{2}+\langle a\rangle(u(t), u(t)) \\
& \leq|\mathcal{T} u(t)|_{L^{2}\left(\mathbb{R}^{m}\right)}^{2}+\left|\dot{u}_{\mathrm{in}}\right|_{L^{2}\left(\mathbb{R}^{m}\right)}^{2}+\left|\mathcal{T} \dot{u}_{\mathrm{in}}\right|_{L^{2}\left(\mathbb{R}^{m}\right)}^{2}+|D|_{H_{Q}^{\infty}}\left|\nabla u_{\mathrm{in}}\right|_{X_{P}}^{2} .
\end{aligned}
$$

It remains to observe that

$$
u(t)=u_{\text {in }}+\int_{0}^{t} \partial_{t} u(s) \mathrm{d} s \text { in } \operatorname{dom} \mathcal{T}
$$

and therefore

$$
\begin{aligned}
|u(t)|_{\text {dom } \mathcal{T}}^{2} & \leq 2\left|u_{\text {in }}\right|_{\text {dom } \mathcal{T}}^{2}+2 t \int_{0}^{t}\left|\partial_{t} u(s)\right|_{\text {dom } \mathcal{T}}^{2} \mathrm{~d} s \\
& \leq 2\left|u_{\text {in }}\right|_{\text {dom } \mathcal{T}}^{2}+2 t^{2}\left[\left|\dot{u}_{\text {in }}\right|_{\text {dom } \mathcal{T}}^{2}+|D|_{H_{Q}^{\infty}}\left|\nabla u_{\text {in }}\right|_{X_{P}}^{2}\right]
\end{aligned}
$$

Finally one gets for any $t \in \mathbb{R}_{+}$

$$
\begin{aligned}
d|u(t)|_{H_{P}^{1}}^{2} & \leq \max \{1, d\}|u(t)|_{\text {dom } \mathcal{T}}^{2}+\left|\dot{u}_{\text {in }}\right|_{\text {dom } \mathcal{T}}^{2}+|D|_{H_{Q}^{\infty}}\left|\nabla u_{\text {in }}\right|_{X_{P}}^{2} \\
& \leq \max \{1, d\}\left[2\left|u_{\text {in }}\right|_{\text {dom } \mathcal{T}}^{2}+2 t^{2}\left(\left|\dot{u}_{\text {in }}\right|_{\text {dom } \mathcal{T}}^{2}+|D|_{H_{Q}^{\infty}}\left|\nabla u_{\text {in }}\right|_{X_{P}}^{2}\right)\right] \\
& +\left|\dot{u}_{\text {in }}\right|_{\text {dom } \mathcal{T}}^{2}+|D|_{H_{Q}^{\infty}}\left|\nabla u_{\text {in }}\right|_{X_{P}}^{2}
\end{aligned}
$$

and we are done because the embedding $H_{P}^{1} \hookrightarrow \operatorname{dom} \mathcal{T}$ is continuous cf. Remark 3.1.

We claim that if $\left(u, \partial_{t} u\right) \in L_{\text {loc }}^{\infty}\left(\mathbb{R}_{+} ; H_{P}^{1}\right) \times L_{\text {loc }}^{\infty}\left(\mathbb{R}_{+} ; \operatorname{dom} \mathcal{T}\right)$ is the solution of (11), (12) associated to the initial conditions $\left(u_{\text {in }}, \dot{u}_{\text {in }}\right) \in H_{P}^{1} \times \operatorname{dom} \mathcal{T}$, then $\left(u_{s},\left(\partial_{t} u\right)_{s}\right) \in$ $L_{\text {loc }}^{\infty}\left(\mathbb{R}_{+} ; H_{P}^{1}\right) \times L_{\text {loc }}^{\infty}\left(\mathbb{R}_{+} ; \operatorname{dom} \mathcal{T}\right)$ is the solution of (11), (12) associated to the initial conditions $\left(\left(u_{\text {in }}\right)_{s},\left(\dot{u}_{\text {in }}\right)_{s}\right) \in H_{P}^{1} \times \operatorname{dom} \mathcal{T}$. Indeed, for any $v \in H_{P}^{1}$ we have

$\frac{\mathrm{d}}{\mathrm{d} t}\left(u(t)_{s}, v\right)_{\operatorname{dom} \mathcal{T}}=\frac{\mathrm{d}}{\mathrm{d} t}\left(u(t), v_{-s}\right)_{\operatorname{dom} \mathcal{T}}=\left(\partial_{t} u(t), v_{-s}\right)_{\operatorname{dom} \mathcal{T}}=\left(\left(\partial_{t} u(t)\right)_{s}, v\right)_{\operatorname{dom} \mathcal{T}}$ in $\mathcal{D}^{\prime}\left(\mathbb{R}_{+}\right)$ saying that $\partial_{t} u_{s}=\left(\partial_{t} u\right)_{s} \in L_{\text {loc }}^{\infty}\left(\mathbb{R}_{+} ; \operatorname{dom} \mathcal{T}\right)$. Moreover, for any $v \in H_{P}^{1}$ we have

$$
\begin{aligned}
& \frac{\mathrm{d}}{\mathrm{d} t}\left(\left(\partial_{t} u(t)\right)_{s}, v\right)_{\operatorname{dom} \mathcal{T}}+\int_{\mathbb{R}^{m}}\langle D\rangle \nabla u(t)_{s} \cdot \nabla v \mathrm{~d} y \\
& \quad=\frac{\mathrm{d}}{\mathrm{d} t}\left(\left(\partial_{t} u(t)\right), v_{-s}\right)_{\operatorname{dom} \mathcal{T}}+\int_{\mathbb{R}^{m}}\langle D\rangle{ }^{t} \partial Y(s ; y)(\nabla u(t))_{s} \cdot{ }^{t} \partial Y(s ; y)\left(\nabla v_{-s}\right)_{s} \mathrm{~d} y \\
& \quad=\frac{\mathrm{d}}{\mathrm{d} t}\left(\left(\partial_{t} u(t)\right), v_{-s}\right)_{\operatorname{dom} \mathcal{T}}+\int_{\mathbb{R}^{m}} \partial Y(s ; y)\langle D\rangle(y){ }^{t} \partial Y(s ; y)(\nabla u(t))_{s} \cdot\left(\nabla v_{-s}\right)_{s} \mathrm{~d} y \\
& \quad=\frac{\mathrm{d}}{\mathrm{d} t}\left(\left(\partial_{t} u(t)\right), v_{-s}\right)_{\operatorname{dom} \mathcal{T}}+\int_{\mathbb{R}^{m}} G(-s)\langle D\rangle \nabla u(t) \cdot \nabla v_{-s} \mathrm{~d} y \\
& \quad=\frac{\mathrm{d}}{\mathrm{d} t}\left(\left(\partial_{t} u(t)\right), v_{-s}\right)_{\operatorname{dom} \mathcal{T}}+\langle a\rangle\left(u(t), v_{-s}\right)=0
\end{aligned}
$$

since $v_{-s} \in H_{P}^{1}$. Therefore $\left(u_{s},\left(\partial_{t} u\right)_{s}\right)$ is the solution of (11), (12) corresponding to the initial condition $\left(\left(u_{\text {in }}\right)_{s},\left(\dot{u}_{\text {in }}\right)_{s}\right)$. In particular, if $\left(u_{\text {in }}, \dot{u}_{\text {in }}\right) \in\left(H_{P}^{1} \cap \operatorname{ker} \mathcal{T}\right) \times \operatorname{ker} \mathcal{T}$, then $\left(\left(u_{\text {in }}\right)_{s},\left(\dot{u}_{\text {in }}\right)_{s}\right)=\left(u_{\text {in }}, \dot{u}_{\text {in }}\right)$ and by the uniqueness of the solution, one gets $\left(u_{s},\left(\partial_{t} u\right)_{s}\right)=$ $\left(u, \partial_{t} u\right)$, saying that $\left(u, \partial_{t} u\right) \in\left(H_{P}^{1} \cap \operatorname{ker} \mathcal{T}\right) \times \operatorname{ker} \mathcal{T}$. 


\section{Asymptotic behavior}

We investigate the be havior of the family of solutions $\left(u^{\varepsilon}\right)_{\varepsilon}$ when $\varepsilon$ becomes small. We assume that the energy of the initial conditions are uniformly bounded, that is

$$
\sup _{0<\varepsilon \leq 1}\left\{\left|u_{\text {in }}^{\varepsilon}\right|_{H_{P}^{1}}+\left|\dot{u}_{\text {in }}^{\varepsilon}\right|_{L^{2}\left(\mathbb{R}^{m}\right)}+\frac{\left|b \cdot \nabla u_{\text {in }}^{\varepsilon}\right|_{L^{2}\left(\mathbb{R}^{m}\right)}}{\varepsilon}\right\}<+\infty .
$$

In particular we deduce that the limit $u$ when $\varepsilon \searrow 0$, of the family $\left(u^{\varepsilon}\right)_{\varepsilon}$, satisfies the constraint $b \cdot \nabla u=0$. When (14) is not verified, oscillations in time may appear, that is $u^{\varepsilon}(t, y) \approx u(t, t / \varepsilon, y)$, where the profile $u$ satisfies the constraint

$$
\partial_{s}^{2} u-\operatorname{div}_{y}(b(y) \otimes b(y) \nabla u)=0, \quad(s, y) \in \mathbb{R} \times \mathbb{R}^{m} .
$$

In this study we neglect the time oscillations and assume that (14) holds true. Appealing to compactness arguments, we prove that the limit, when $\varepsilon \searrow 0$, of the family of solutions $\left(u^{\varepsilon}\right)_{\varepsilon}$ solves a wave problem as well.

\section{Theorem 4.1}

Assume that (3), (4), (5), (6), (7), (9), (10) hold true and that the initial conditions satisfy

$$
\begin{gathered}
\lim _{\varepsilon \searrow 0} u_{\text {in }}^{\varepsilon}=u_{\text {in }} \quad \text { weakly in } H_{P}^{1} \\
\lim _{\varepsilon \searrow 0} \dot{u}_{\text {in }}^{\varepsilon}=\dot{u}_{\text {in }} \quad \text { weakly in } L^{2}\left(\mathbb{R}^{m}\right)
\end{gathered}
$$

and

$$
\sup _{0<\varepsilon \leq 1} \frac{\left|b \cdot \nabla u_{\text {in }}^{\varepsilon}\right|_{L^{2}\left(\mathbb{R}^{m}\right)}}{\varepsilon}<+\infty .
$$

Then $\left(u^{\varepsilon}\right)_{\varepsilon},\left(\partial_{t} u^{\varepsilon}\right)_{\varepsilon}$ converge weakly $\star$ in $L^{\infty}\left([0, T], H_{P}^{1}\right), L^{\infty}\left([0, T] ; L^{2}\left(\mathbb{R}^{m}\right)\right)$ respectively, $T \in \mathbb{R}_{+}$, toward the solution $\left(u, \partial_{t} u\right) \in L_{\mathrm{loc}}^{\infty}\left(\mathbb{R}_{+} ; H_{P}^{1} \cap \operatorname{ker} \mathcal{T}\right) \times L_{\mathrm{loc}}^{\infty}\left(\mathbb{R}_{+} ; \operatorname{ker} \mathcal{T}\right)$ of the problem

$$
\frac{\mathrm{d}}{\mathrm{d} t} \int_{\mathbb{R}^{m}} \partial_{t} u v \mathrm{~d} y+\int_{\mathbb{R}^{m}}\langle D\rangle(y) \nabla u \cdot \nabla v \mathrm{~d} y=0 \quad \text { in } \mathcal{D}^{\prime}\left(\mathbb{R}_{+}\right), v \in H_{P}^{1}
$$

with $\left(u(0), \partial_{t} u(0)\right)=\left(u_{\text {in }},\left\langle\dot{u}_{\text {in }}\right\rangle\right)$.

Proof. We have

$$
\sup _{\varepsilon>0}\left\{\left|u_{\text {in }}^{\varepsilon}\right|_{H_{P}^{1}}+\left|\dot{u}_{\text {in }}^{\varepsilon}\right|_{L^{2}\left(\mathbb{R}^{m}\right)}+\frac{1}{\varepsilon}\left|b \cdot \nabla u_{\text {in }}^{\varepsilon}\right|_{L^{2}\left(\mathbb{R}^{m}\right)}\right\}<+\infty
$$

and therefore, by Proposition 3.2, there is a sequence $\left.\left.\left(\varepsilon_{k}\right)_{k} \subset\right] 0,1\right], \lim _{k \rightarrow+\infty} \varepsilon_{k}=0$, and two functions $u \in L_{\text {loc }}^{\infty}\left(\mathbb{R}_{+} ; H_{P}^{1}\right), \dot{u} \in L_{\text {loc }}^{\infty}\left(\mathbb{R}_{+} ; L^{2}\left(\mathbb{R}^{m}\right)\right)$ such that, for any $T \in \mathbb{R}_{+}$ we have

$$
\begin{gathered}
u^{\varepsilon_{k}} \rightarrow u \text { weakly } \star \text { in } L^{\infty}\left([0, T] ; H_{P}^{1}\right) \\
\partial_{t} u^{\varepsilon_{k}} \rightarrow \dot{u} \text { weakly } \star \text { in } L^{\infty}\left([0, T] ; L^{2}\left(\mathbb{R}^{m}\right)\right) .
\end{gathered}
$$


For any $v \in L^{2}\left(\mathbb{R}^{m}\right)$ and $\eta \in C_{c}^{1}\left(\mathbb{R}_{+}\right)$we have

$$
\eta(0)\left(u_{\mathrm{in}}^{\varepsilon_{k}}, v\right)_{L^{2}\left(\mathbb{R}^{m}\right)}+\int_{\mathbb{R}_{+}} \eta(t)\left(\partial_{t} u^{\varepsilon_{k}}(t), v\right)_{L^{2}\left(\mathbb{R}^{m}\right)} \mathrm{d} t+\int_{\mathbb{R}_{+}} \eta^{\prime}(t)\left(u^{\varepsilon_{k}}(t), v\right)_{L^{2}\left(\mathbb{R}^{m}\right)} \mathrm{d} t=0 .
$$

Passing to the limit when $k \rightarrow+\infty$, yields

$$
\eta(0)\left(u_{\mathrm{in}}, v\right)_{L^{2}\left(\mathbb{R}^{m}\right)}+\int_{\mathbb{R}_{+}} \eta(t)(\dot{u}(t), v)_{L^{2}\left(\mathbb{R}^{m}\right)} \mathrm{d} t+\int_{\mathbb{R}_{+}} \eta^{\prime}(t)(u(t), v)_{L^{2}\left(\mathbb{R}^{m}\right)} \mathrm{d} t=0
$$

saying that $\dot{u}$ is the time derivative of $u$

$$
u(0)=u_{\text {in }}, \quad \frac{\mathrm{d}}{\mathrm{d} t}(u(t), v)_{L^{2}\left(\mathbb{R}^{m}\right)}=(\dot{u}(t), v)_{L^{2}\left(\mathbb{R}^{m}\right)} \quad \text { in } \mathcal{D}^{\prime}\left(\mathbb{R}_{+}\right) .
$$

We claim that $b \cdot \nabla u=0$. Indeed, we have, thanks to the estimate in Proposition 3.2

$$
\lim _{k \rightarrow+\infty}\left|b \cdot \nabla u^{\varepsilon_{k}}\right|_{C\left([0, T] ; L^{2}\left(\mathbb{R}^{m}\right)\right)}=0
$$

and thus, for any $\eta \in C_{c}\left(\mathbb{R}_{+}\right)$and $\varphi \in C_{c}^{1}\left(\mathbb{R}^{m}\right)$ we obtain

$$
\int_{\mathbb{R}_{+}} \eta(t) \int_{\mathbb{R}^{m}} b \cdot \nabla \varphi u^{\varepsilon_{k}}(t, y) \mathrm{d} y \mathrm{~d} t=-\int_{\mathbb{R}_{+}} \eta(t) \int_{\mathbb{R}^{m}} \varphi(y) b \cdot \nabla u^{\varepsilon_{k}}(t) \mathrm{d} y \mathrm{~d} t \rightarrow 0 \text { as } k \rightarrow+\infty
$$

By the convergence

$$
\lim _{k \rightarrow+\infty} \int_{\mathbb{R}_{+}} \eta(t) \int_{\mathbb{R}^{m}} b \cdot \nabla \varphi u^{\varepsilon_{k}}(t, y) \mathrm{d} y \mathrm{~d} t=\int_{\mathbb{R}_{+}} \eta(t) \int_{\mathbb{R}^{m}} b \cdot \nabla \varphi u(t, y) \mathrm{d} y \mathrm{~d} t
$$

we deduce that

$$
\int_{\mathbb{R}^{m}} b \cdot \nabla \varphi u(t, y) \mathrm{d} y=0, \quad \text { for a.a. } t \in \mathbb{R}_{+} .
$$

As $t \in \mathbb{R}_{+} \mapsto u(t) \in L^{2}\left(\mathbb{R}^{m}\right)$ is continuous (since $\partial_{t} u=\dot{u} \in L_{\text {loc }}^{\infty}\left(\mathbb{R}_{+} ; L^{2}\left(\mathbb{R}^{m}\right)\right)$ ), the previous equality holds true for any $t \in \mathbb{R}_{+}$and $\varphi \in C_{c}^{1}\left(\mathbb{R}^{m}\right)$ saying that $b \cdot \nabla u=0$. In particular we have $b \cdot \nabla u_{\text {in }}=0$. Notice also that for any $\varphi \in C_{c}^{1}\left(\mathbb{R}^{m}\right)$ we can write

$$
\int_{\mathbb{R}^{m}} \partial_{t} u b \cdot \nabla \varphi \mathrm{d} y=\frac{\mathrm{d}}{\mathrm{d} t} \int_{\mathbb{R}^{m}} u b \cdot \nabla \varphi \mathrm{d} y=0, \quad \text { in } \mathcal{D}^{\prime}\left(\mathbb{R}_{+}\right)
$$

implying that $\partial_{t} u \in \operatorname{dom} \mathcal{T}, \mathcal{T} \partial_{t} u=0$.

We appeal now to the variational formulation of (1), (2) with $v \in H_{P}^{1} \cap \operatorname{ker} \mathcal{T}$

$$
\frac{\mathrm{d}}{\mathrm{d} t} \int_{\mathbb{R}^{m}} \partial_{t} u^{\varepsilon_{k}} v(y) \mathrm{d} y+\int_{\mathbb{R}^{m}} D(y) \nabla u^{\varepsilon_{k}}(t) \cdot \nabla v \mathrm{~d} y=0 \quad \text { in } \mathcal{D}^{\prime}\left(\mathbb{R}_{+}\right) .
$$

For any $\eta \in C_{c}^{1}([0, T[)$ we write

$$
-\eta(0) \int_{\mathbb{R}^{m}} \dot{u}_{\text {in }}^{\varepsilon_{k}} v \mathrm{~d} y-\int_{0}^{T} \eta^{\prime}(t) \int_{\mathbb{R}^{m}} \partial_{t} u^{\varepsilon_{k}} v \mathrm{~d} y \mathrm{~d} t+\int_{0}^{T} \eta(t) \int_{\mathbb{R}^{m}} D(y) \nabla u^{\varepsilon_{k}}(t) \cdot \nabla v \mathrm{~d} y \mathrm{~d} t=0 .
$$


Thanks to the convergences of $\left(\dot{u}_{\text {in }}^{\varepsilon_{k}}\right)_{k}$ weakly in $L^{2}\left(\mathbb{R}^{m}\right)$, of $\left(\partial_{t} u^{\varepsilon_{k}}\right)_{k}$ weakly $\star$ in $L^{\infty}\left([0, T] ; L^{2}\right)$ and of $\left(u^{\varepsilon_{k}}\right)_{k}$ weakly $\star$ in $L^{\infty}\left([0, T] ; H_{P}^{1}\right)$ we deduce for any $v \in H_{P}^{1} \cap \operatorname{ker} \mathcal{T}$

$$
-\eta(0) \int_{\mathbb{R}^{m}} \dot{u}_{\text {in }} v \mathrm{~d} y-\int_{0}^{T} \eta^{\prime}(t) \int_{\mathbb{R}^{m}} \partial_{t} u v \mathrm{~d} y \mathrm{~d} t+\int_{0}^{T} \eta(t) \int_{\mathbb{R}^{m}} D(y) \nabla u(t) \cdot \nabla v \mathrm{~d} y \mathrm{~d} t=0 .
$$

Clearly we have

$$
\eta(0) \int_{\mathbb{R}^{m}} \dot{u}_{\text {in }}(y) v(y) \mathrm{d} y=\eta(0) \int_{\mathbb{R}^{m}}\left\langle\dot{u}_{\text {in }}\right\rangle(y) v(y) \mathrm{d} y
$$

We concentrate now on the term $\int_{\mathbb{R}^{m}} D(y) \nabla u(t) \cdot \nabla v \mathrm{~d} y$. We know that $u(t), v \in$ $H_{P}^{1} \cap \operatorname{ker} \mathcal{T}$ and therefore we can write for any $s \in[0, S], S \in \mathbb{R}_{+}$

$$
\begin{aligned}
\int_{\mathbb{R}^{m}} D(y) \nabla u(t) & \cdot \nabla v \mathrm{~d} y=\int_{\mathbb{R}^{m}} D(y) \nabla(u(t))_{-s} \cdot \nabla v_{-s} \mathrm{~d} y \\
& =\int_{\mathbb{R}^{m}} D(y)^{t} \partial Y(-s ; y)(\nabla u(t))_{-s} \cdot{ }^{t} \partial Y(-s ; y)(\nabla v)_{-s} \mathrm{~d} y \\
& =\int_{\mathbb{R}^{m}} \partial Y(-s ; y) D(y)^{t} \partial Y(-s ; y)(\nabla u(t))_{-s} \cdot(\nabla v)_{-s} \mathrm{~d} y \\
& =\int_{\mathbb{R}^{m}} \partial Y(-s ; Y(s ; z)) D(Y(s ; z))^{t} \partial Y(-s ; Y(s ; z)) \nabla u(t, z) \cdot \nabla v(z) \mathrm{d} z \\
& =\int_{\mathbb{R}^{m}} G(s) D \nabla u(t) \cdot \nabla v \mathrm{~d} y .
\end{aligned}
$$

Averaging with respect to $s$ we obtain

$$
\int_{\mathbb{R}^{m}} D(y) \nabla u(t) \cdot \nabla v \mathrm{~d} y=\int_{\mathbb{R}^{m}} \frac{1}{S} \int_{0}^{S} G(s) D \mathrm{~d} s \nabla u(t) \cdot \nabla v \mathrm{~d} y .
$$

As $\nabla u(t) \in X_{P}$, we have by Lemma 2.1 the convergence

$$
\lim _{S \rightarrow+\infty} \frac{1}{S} \int_{0}^{S} G(s) D \nabla u(t) \mathrm{d} s=\langle D\rangle \nabla u(t), \quad \text { in } X_{Q} .
$$

Since $\nabla v \in X_{P}$ we obtain

$$
\lim _{S \rightarrow+\infty} \int_{\mathbb{R}^{m}} \frac{1}{S} \int_{0}^{S} G(s) D \mathrm{~d} s \nabla u(t) \cdot \nabla v \mathrm{~d} y=\int_{\mathbb{R}^{m}}\langle D\rangle \nabla u(t) \cdot \nabla v \mathrm{~d} y
$$

and thus we have

$$
\int_{\mathbb{R}^{m}} D(y) \nabla u(t) \cdot \nabla v \mathrm{~d} y=\int_{\mathbb{R}^{m}}\langle D\rangle(y) \nabla u(t) \cdot \nabla v \mathrm{~d} y, \quad v \in H_{P}^{1} \cap \operatorname{ker} \mathcal{T} .
$$

Coming back in (15) we deduce

$$
-\eta(0) \int_{\mathbb{R}^{m}}\left\langle\dot{u}_{\text {in }}\right\rangle v \mathrm{~d} y-\int_{0}^{T} \eta^{\prime}(t) \int_{\mathbb{R}^{m}} \partial_{t} u v \mathrm{~d} y \mathrm{~d} t+\int_{0}^{T} \eta(t) \int_{\mathbb{R}^{m}}\langle D\rangle \nabla u(t) \cdot \nabla v \mathrm{~d} y \mathrm{~d} t=0
$$


for any $\eta \in C_{c}^{1}\left(\mathbb{R}_{+}\right), v \in H_{P}^{1} \cap \operatorname{ker} \mathcal{T}$. Actually, the above formulation holds true for any $v \in H_{P}^{1}$. Indeed, for any $v \in H_{P}^{1}$, we have $v=v_{1}+v_{2}, v_{1}=v-\langle v\rangle \in H_{P}^{1}, v_{2}=$ $\langle v\rangle \in H_{P}^{1} \cap \operatorname{ker} \mathcal{T}$, cf. Proposition 2.5. Clearly, (16) is satisfied when considering $v_{2}$. We are done if we justify that (16) holds true when taking $v_{1} \in H_{P}^{1},\left\langle v_{1}\right\rangle=0$. We have

$$
\eta(0) \int_{\mathbb{R}^{m}}\left\langle\dot{u}_{\text {in }}\right\rangle(y) v_{1}(y) \mathrm{d} y=\eta(0) \int_{\mathbb{R}^{m}}\left\langle\dot{u}_{\text {in }}\right\rangle(y)\left\langle v_{1}\right\rangle(y) \mathrm{d} y=0
$$

and since $\partial_{t} u \in \operatorname{ker} \mathcal{T}$ we can write

$$
\int_{0}^{T} \eta^{\prime}(t) \int_{\mathbb{R}^{m}} \partial_{t} u v_{1}(y) \mathrm{d} y \mathrm{~d} t=\int_{0}^{T} \eta^{\prime}(t) \int_{\mathbb{R}^{m}} \partial_{t} u\left\langle v_{1}\right\rangle(y) \mathrm{d} y \mathrm{~d} t=0 .
$$

As before, we have for any $s \in[0, S], S \in \mathbb{R}_{+}$

$$
\begin{aligned}
\int_{\mathbb{R}^{m}}\langle D\rangle \nabla u(t) \cdot \nabla v_{1} \mathrm{~d} y & =\int_{\mathbb{R}^{m}}\langle D\rangle \nabla u(t)_{-s} \cdot \nabla\left(v_{1 s}\right)_{-s} \mathrm{~d} y \\
& =\int_{\mathbb{R}^{m}}\langle D\rangle(y)^{t} \partial Y(-s ; y)(\nabla u(t))_{-s} \cdot{ }^{t} \partial Y(-s ; y)\left(\nabla v_{1 s}\right)_{-s} \mathrm{~d} y \\
& =\int_{\mathbb{R}^{m}} \partial Y(-s ; y)\langle D\rangle(y)^{t} \partial Y(-s ; y)(\nabla u(t))_{-s} \cdot\left(\nabla v_{1 s}\right)_{-s} \mathrm{~d} y \\
& =\int_{\mathbb{R}^{m}} G(s)\langle D\rangle \nabla u(t) \cdot \nabla v_{1 s} \mathrm{~d} y \\
& =\int_{\mathbb{R}^{m}}\langle D\rangle \nabla u(t) \cdot \nabla v_{1 s} \mathrm{~d} y \\
& =\int_{\mathbb{R}^{m}}\langle D\rangle \nabla u(t) \cdot \frac{1}{S} \int_{0}^{T} \nabla v_{1 s} \mathrm{~d} s \mathrm{~d} y .
\end{aligned}
$$

By Proposition 2.5 we know that

$$
\lim _{S \rightarrow+\infty} \frac{1}{S} \int_{0}^{S} \nabla v_{1 s} \mathrm{~d} s=\nabla\left\langle v_{1}\right\rangle=0 \text { strongly in } X_{P} .
$$

Notice that $\langle D\rangle \nabla u(t)$ belongs to $X_{Q}$

$$
|\langle D\rangle \nabla u(t)|_{X_{Q}} \leq|\langle D\rangle|_{H_{Q}^{\infty}}|\nabla u(t)|_{X_{P}} \leq|D|_{H_{Q}^{\infty}}|\nabla u(t)|_{X_{P}}
$$

and therefore we deduce

$$
\lim _{S \rightarrow+\infty} \int_{\mathbb{R}^{m}}\langle D\rangle \nabla u(t) \cdot \frac{1}{S} \int_{0}^{S} \nabla v_{1 s} \mathrm{~d} s \mathrm{~d} y=0
$$

implying that $\int_{\mathbb{R}^{m}}\langle D\rangle \nabla u(t) \cdot \nabla v_{1} \mathrm{~d} y=0$. Finally (16) is trivially satisfied for any $v_{1} \in H_{P}^{1},\left\langle v_{1}\right\rangle=0$, and thus for any $v \in H_{P}^{1}$ we have

$$
\frac{\mathrm{d}}{\mathrm{d} t} \int_{\mathbb{R}^{m}} \partial_{t} u v(y) \mathrm{d} y+\int_{\mathbb{R}^{m}}\langle D\rangle(y) \nabla u(t) \cdot \nabla v \mathrm{~d} y=0 \quad \text { in } \mathcal{D}^{\prime}\left(\mathbb{R}_{+}\right)
$$

and $\left(u(0), \partial_{t} u(0)\right)=\left(u_{\text {in }},\left\langle\dot{u}_{\text {in }}\right\rangle\right)$. The convergence of all the family $\left(u^{\varepsilon}, \partial_{t} u^{\varepsilon}\right)_{\varepsilon}$ follows by the uniqueness of the solution of the above variational formulation. 
We investigate now strong convergence results. It happens that the previous weak $\star$ convergences become strong if the initial conditions are well prepared

$$
\begin{gathered}
\lim _{\varepsilon \searrow 0} u_{\text {in }}^{\varepsilon}=u_{\text {in }} \text { strongly in } H_{P}^{1}, \lim _{\varepsilon \searrow 0} \frac{b \cdot \nabla u_{\text {in }}^{\varepsilon}}{\varepsilon}=0 \text { strongly in } L^{2}\left(\mathbb{R}^{m}\right) \\
\lim _{\varepsilon \searrow 0} \dot{u}_{\text {in }}^{\varepsilon}=\dot{u}_{\text {in }} \text { strongly in } L^{2}\left(\mathbb{R}^{m}\right), \quad b \cdot \nabla \dot{u}_{\text {in }}=0 .
\end{gathered}
$$

We are using the following easy lemma.

\section{Lemma 4.1}

Let $\left(A^{\varepsilon}\right)_{\varepsilon>0},\left(B^{\varepsilon}\right)_{\varepsilon>0}$ be two families of real numbers and $A, B \in \mathbb{R}$ such that

$$
\limsup _{\varepsilon \searrow 0}\left(A^{\varepsilon}+B^{\varepsilon}\right) \leq A+B, \quad A \leq \liminf _{\varepsilon \searrow 0} A^{\varepsilon}, \quad B \leq \liminf _{\varepsilon \searrow 0} B^{\varepsilon} .
$$

Then we have

$$
\lim _{\varepsilon \searrow 0} A^{\varepsilon}=A, \lim _{\varepsilon \searrow 0} B^{\varepsilon}=B .
$$

Proof. We have the inequalities

$$
A+B \geq \limsup _{\varepsilon \searrow 0}\left(A^{\varepsilon}+B^{\varepsilon}\right) \geq \limsup _{\varepsilon \searrow 0} A^{\varepsilon}+\liminf _{\varepsilon \searrow 0} B^{\varepsilon} \geq \underset{\varepsilon \searrow 0}{\limsup _{\varepsilon}} A^{\varepsilon}+B
$$

saying that

$$
A \leq \liminf _{\varepsilon \searrow 0} A^{\varepsilon} \leq \limsup _{\varepsilon \searrow 0} A^{\varepsilon} \leq A .
$$

Therefore $\left(A^{\varepsilon}\right)_{\varepsilon>0}$ converges toward $A$ as $\varepsilon \searrow 0$. Similarly, $\left(B^{\varepsilon}\right)_{\varepsilon>0}$ converges toward $B$ as $\varepsilon \searrow 0$.

We appeal to the following standard result which allows us to transform weak convergence in strong convergence.

\section{Proposition 4.1}

Assume that $A \in H_{Q}^{\infty}$ and that $\left(w^{\varepsilon}\right)_{\varepsilon}$ converges weakly in $L^{2}\left([0, T] ; X_{P}\right)$ toward $w^{0}$, when $\varepsilon \searrow 0$.

1. If $A=A(y)$ are non-negative, then

$$
\int_{0}^{T} \int_{\mathbb{R}^{m}} A(y) w^{0}(t) \cdot w^{0}(t) \mathrm{d} y \mathrm{~d} t \leq \liminf _{\varepsilon \searrow 0} \int_{0}^{T} \int_{\mathbb{R}^{m}} A(y) w^{\varepsilon}(t) \cdot w^{\varepsilon}(t) \mathrm{d} y \mathrm{~d} t .
$$

2. If there is $d>0$ such that $Q^{1 / 2} A Q^{1 / 2} \geq d I_{m}$ and

$$
\underset{\varepsilon \searrow 0}{\limsup } \int_{0}^{T} \int_{\mathbb{R}^{m}} A(y) w^{\varepsilon}(t) \cdot w^{\varepsilon}(t) \mathrm{d} y \mathrm{~d} t \leq \int_{0}^{T} \int_{\mathbb{R}^{m}} A(y) w^{0}(t) \cdot w^{0}(t) \mathrm{d} y \mathrm{~d} t
$$

then the family $\left(w^{\varepsilon}\right)_{\varepsilon}$ converges strongly in $L^{2}\left([0, T] ; X_{P}\right)$ toward $w^{0}$, when $\varepsilon \searrow 0$. 


\section{Proof.}

1. By the inequality

$$
\left|\int_{\mathbb{R}^{m}} A(y) \xi(y) \cdot \eta(y) \mathrm{d} y\right| \leq|A|_{H_{Q}^{\infty}}|\xi|_{X_{P}}|\eta|_{X_{P}}, \quad \xi, \eta \in X_{P}
$$

we deduce that the bilinear form $(\xi, \eta) \rightarrow \int_{0}^{T} \int_{\mathbb{R}^{m}} A(y) \xi(t, y) \cdot \eta(t, y) \mathrm{d} y \mathrm{~d} t$ is continuous on $L^{2}\left([0, T] ; X_{P}\right) \times L^{2}\left([0, T] ; X_{P}\right)$. The non-negativity of $A$ allows us to write

$$
\begin{aligned}
0 & \leq \int_{0}^{T} \int_{\mathbb{R}^{m}} A(y)\left[w^{\varepsilon}(t, y)-w^{0}(t, y)\right] \cdot\left[w^{\varepsilon}(t, y)-w^{0}(t, y)\right] \mathrm{d} y \mathrm{~d} t \\
& =\int_{0}^{T} \int_{\mathbb{R}^{m}} A(y) w^{\varepsilon}(t, y) \cdot w^{\varepsilon}(t, y) \mathrm{d} y \mathrm{~d} t+\int_{0}^{T} \int_{\mathbb{R}^{m}} A(y) w^{0}(t, y) \cdot w^{0}(t, y) \mathrm{d} y \mathrm{~d} t \\
& -\int_{0}^{T} \int_{\mathbb{R}^{m}} A(y) w^{\varepsilon}(t, y) \cdot w^{0}(t, y) \mathrm{d} y \mathrm{~d} t-\int_{0}^{T} \int_{\mathbb{R}^{m}} A(y) w^{0}(t, y) \cdot w^{\varepsilon}(t, y) \mathrm{d} y \mathrm{~d} t
\end{aligned}
$$

and our conclusion follows immediately by taking $\lim \inf _{\varepsilon \searrow 0}$.

2. In this case, the hypothesis $Q^{1 / 2} A Q^{1 / 2} \geq d I_{m}$ implies

$$
\begin{aligned}
d\left|w^{\varepsilon}-w^{0}\right|_{X_{P}}^{2} & \leq \int_{0}^{T} \int_{\mathbb{R}^{m}} A(y) w^{\varepsilon}(t, y) \cdot w^{\varepsilon}(t, y) \mathrm{d} y \mathrm{~d} t+\int_{0}^{T} \int_{\mathbb{R}^{m}} A(y) w^{0}(t, y) \cdot w^{0}(t, y) \mathrm{d} y \mathrm{~d} t \\
& -\int_{0}^{T} \int_{\mathbb{R}^{m}} A(y) w^{\varepsilon}(t, y) \cdot w^{0}(t, y) \mathrm{d} y \mathrm{~d} t-\int_{0}^{T} \int_{\mathbb{R}^{m}} A(y) w^{0}(t, y) \cdot w^{\varepsilon}(t, y) \mathrm{d} y \mathrm{~d} t .
\end{aligned}
$$

Taking $\lim \sup _{\varepsilon \searrow 0}$ yields

$d \limsup _{\varepsilon \searrow 0}\left|w^{\varepsilon}-w^{0}\right|_{X_{P}}^{2} \leq \limsup _{\varepsilon \searrow 0} \int_{0}^{T} \int_{\mathbb{R}^{m}} A(y) w^{\varepsilon} \cdot w^{\varepsilon} \mathrm{d} y \mathrm{~d} t-\int_{0}^{T} \int_{\mathbb{R}^{m}} A(y) w^{0} \cdot w^{0} \mathrm{~d} y \mathrm{~d} t \leq 0$

saying that the family $\left(w^{\varepsilon}\right)_{\varepsilon>0}$ converges strongly in $L^{2}\left([0, T] ; X_{P}\right)$ toward $w^{0}$ when $\varepsilon \searrow 0$.

We are ready now to improve the asymptotic behavior stated in Theorem 4.1. When the initial conditions are well prepared, we expect strong convergence results.

\section{Theorem 4.2}

Assume that (3), (4), (5), (6), (7), (9), (10) hold true and that the initial conditions satisfy

$$
\begin{gathered}
\lim _{\varepsilon \searrow 0} u_{\text {in }}^{\varepsilon}=u_{\text {in }} \text { strongly in } H_{P}^{1}, \lim _{\varepsilon \searrow 0} \frac{b \cdot \nabla u_{\text {in }}^{\varepsilon}}{\varepsilon}=0 \text { strongly in } L^{2}\left(\mathbb{R}^{m}\right) \\
\lim _{\varepsilon \searrow 0} \dot{u}_{\text {in }}^{\varepsilon}=\dot{u}_{\text {in }} \text { strongly in } L^{2}\left(\mathbb{R}^{m}\right), \quad b \cdot \nabla \dot{u}_{\text {in }}=0 .
\end{gathered}
$$

Then we have the strong convergences

$$
\begin{gathered}
\lim _{\varepsilon \searrow 0} u^{\varepsilon}=u \quad \text { in } L_{\mathrm{loc}}^{\infty}\left(\mathbb{R}_{+} ; L^{2}\left(\mathbb{R}^{m}\right)\right), \lim _{\varepsilon \searrow 0} \nabla u^{\varepsilon}=\nabla u \text { in } L_{\mathrm{loc}}^{2}\left(\mathbb{R}_{+} ; X_{P}\right) \\
\lim _{\varepsilon \searrow 0} \partial_{t} u^{\varepsilon}=\partial_{t} u \text { in } L_{\mathrm{loc}}^{2}\left(\mathbb{R}_{+} ; L^{2}\left(\mathbb{R}^{m}\right)\right), \lim _{\varepsilon \searrow 0} \frac{b \cdot \nabla u^{\varepsilon}}{\varepsilon}=0 \quad \text { in } L_{\mathrm{loc}}^{2}\left(\mathbb{R}_{+} ; L^{2}\left(\mathbb{R}^{m}\right)\right) .
\end{gathered}
$$


Proof. The arguments rely on the energy conservations

$$
\begin{aligned}
& \left|\partial_{t} u^{\varepsilon}(t)\right|_{L^{2}\left(\mathbb{R}^{m}\right)}^{2}+\int_{\mathbb{R}^{m}} D(y) \nabla u^{\varepsilon}(t) \cdot \nabla u^{\varepsilon}(t) \mathrm{d} y+\frac{1}{\varepsilon^{2}}\left|b \cdot \nabla u^{\varepsilon}(t)\right|_{L^{2}\left(\mathbb{R}^{m}\right)}^{2} \\
& =\left|\dot{u}_{\mathrm{in}}^{\varepsilon}\right|_{L^{2}\left(\mathbb{R}^{m}\right)}^{2}+\int_{\mathbb{R}^{m}} D(y) \nabla u_{\mathrm{in}}^{\varepsilon} \cdot \nabla u_{\mathrm{in}}^{\varepsilon} \mathrm{d} y+\frac{1}{\varepsilon^{2}}\left|b \cdot \nabla u_{\mathrm{in}}^{\varepsilon}\right|_{L^{2}\left(\mathbb{R}^{m}\right)}^{2}, \quad t \in \mathbb{R}_{+}, \quad 0<\varepsilon \leq 1
\end{aligned}
$$

and

$\left|\partial_{t} u(t)\right|_{L^{2}\left(\mathbb{R}^{m}\right)}^{2}+\int_{\mathbb{R}^{m}}\langle D\rangle(y) \nabla u(t) \cdot \nabla u(t) \mathrm{d} y=\left|\left\langle\dot{u}_{\mathrm{in}}\right\rangle\right|_{L^{2}\left(\mathbb{R}^{m}\right)}^{2}+\int_{\mathbb{R}^{m}}\langle D\rangle(y) \nabla u_{\mathrm{in}} \cdot \nabla u_{\mathrm{in}} \mathrm{d} y$.

As $b \cdot \nabla u=0$, we have already seen (cf. proof of Theorem 4.1) that

$$
\int_{\mathbb{R}^{m}}\langle D\rangle(y) \nabla u(t) \cdot \nabla u(t) \mathrm{d} y=\int_{\mathbb{R}^{m}} D(y) \nabla u(t) \cdot \nabla u(t) \mathrm{d} y, \quad t \in \mathbb{R}_{+}
$$

and thus, for any $T \in \mathbb{R}_{+}$we write

$$
\begin{aligned}
& \int_{0}^{T}\left|\partial_{t} u^{\varepsilon}(t)\right|_{L^{2}\left(\mathbb{R}^{m}\right)}^{2} \mathrm{~d} t+\int_{0}^{T} \int_{\mathbb{R}^{m}}(D(y)+b(y) \otimes b(y)) \nabla u^{\varepsilon}(t) \cdot \nabla u^{\varepsilon}(t) \mathrm{d} y \mathrm{~d} t \\
& =\int_{0}^{T}\left|\partial_{t} u(t)\right|_{L^{2}\left(\mathbb{R}^{m}\right)}^{2} \mathrm{~d} t+\int_{0}^{T} \int_{\mathbb{R}^{m}}(D(y)+b(y) \otimes b(y)) \nabla u(t) \cdot \nabla u(t) \mathrm{d} y \mathrm{~d} t \\
& +T\left[\left|\dot{u}_{\mathrm{in}}\right|_{L^{2}\left(\mathbb{R}^{m}\right)}^{2}-\left|\left\langle\dot{u}_{\text {in }}\right\rangle\right|_{L^{2}\left(\mathbb{R}^{m}\right)}^{2}\right] \\
& +T\left(\int_{\mathbb{R}^{m}}(D(y)+b(y) \otimes b(y)) \nabla u_{\mathrm{in}}^{\varepsilon} \cdot \nabla u_{\mathrm{in}}^{\varepsilon} \mathrm{d} y-\int_{\mathbb{R}^{m}}(D(y)+b(y) \otimes b(y)) \nabla u_{\text {in }} \cdot \nabla u_{\text {in }} \mathrm{d} y\right) \\
& +T\left(\frac{1}{\varepsilon^{2}}-1\right)\left|b \cdot \nabla u_{\mathrm{in}}^{\varepsilon}\right|_{L^{2}\left(\mathbb{R}^{m}\right)}^{2}-\left(\frac{1}{\varepsilon^{2}}-1\right) \int_{0}^{T}\left|b \cdot \nabla u^{\varepsilon}(t)\right|_{L^{2}\left(\mathbb{R}^{m}\right)}^{2} \mathrm{~d} t .
\end{aligned}
$$

We deduce that

$$
\begin{aligned}
\underset{\varepsilon \searrow 0}{\limsup } & {\left[\int_{0}^{T}\left|\partial_{t} u^{\varepsilon}(t)\right|_{L^{2}\left(\mathbb{R}^{m}\right)}^{2} \mathrm{~d} t+\int_{0}^{T} \int_{\mathbb{R}^{m}}(D(y)+b(y) \otimes b(y)) \nabla u^{\varepsilon}(t) \cdot \nabla u^{\varepsilon}(t) \mathrm{d} y \mathrm{~d} t\right] } \\
\leq & \int_{0}^{T}\left|\partial_{t} u(t)\right|_{L^{2}\left(\mathbb{R}^{m}\right)}^{2} \mathrm{~d} t+\int_{0}^{T} \int_{\mathbb{R}^{m}}(D(y)+b(y) \otimes b(y)) \nabla u(t) \cdot \nabla u(t) \mathrm{d} y \mathrm{~d} t .
\end{aligned}
$$

The weak $\star$ convergences of $\left(u^{\varepsilon}\right)_{\varepsilon},\left(\partial_{t} u^{\varepsilon}\right)_{\varepsilon}$ in $L^{\infty}\left([0, T] ; H_{P}^{1}\right), L^{\infty}\left([0, T] ; L^{2}\left(\mathbb{R}^{m}\right)\right)$ respectively, imply the weak convergences of $\left(u^{\varepsilon}\right)_{\varepsilon},\left(\partial_{t} u^{\varepsilon}\right)_{\varepsilon}$ in $L^{2}\left([0, T] ; H_{P}^{1}\right), L^{2}\left([0, T] ; L^{2}\left(\mathbb{R}^{m}\right)\right)$ respectively and therefore

$$
\begin{gathered}
\partial_{t} u^{\varepsilon} \rightarrow \partial_{t} u \text { weakly in } L^{2}\left([0, T] ; L^{2}\left(\mathbb{R}^{m}\right)\right) \text { as } \varepsilon \searrow 0 \\
\nabla u^{\varepsilon} \rightarrow \nabla u \text { weakly in } L^{2}\left([0, T] ; X_{P}\right) \text { as } \varepsilon \searrow 0 .
\end{gathered}
$$

We have, thanks to the first statement in Proposition 4.1, applied with $w^{\varepsilon}=\nabla u^{\varepsilon}$ and $A=D+b \otimes b,|A|_{H_{Q}^{\infty}} \leq|D|_{H_{Q}^{\infty}}+|b|_{X_{Q}^{\infty}}^{2}$

$$
\int_{0}^{T}\left|\partial_{t} u(t)\right|_{L^{2}\left(\mathbb{R}^{m}\right)}^{2} \mathrm{~d} t \leq \liminf _{\varepsilon \searrow 0} \int_{0}^{T}\left|\partial_{t} u^{\varepsilon}(t)\right|_{L^{2}\left(\mathbb{R}^{m}\right)}^{2} \mathrm{~d} t
$$


$\int_{0}^{T} \int_{\mathbb{R}^{m}}(D+b \otimes b) \nabla u(t) \cdot \nabla u(t) \mathrm{d} y \mathrm{~d} t \leq \liminf _{\varepsilon \searrow 0} \int_{0}^{T} \int_{\mathbb{R}^{m}}(D+b \otimes b) \nabla u^{\varepsilon}(t) \cdot \nabla u^{\varepsilon}(t) \mathrm{d} y \mathrm{~d} t$. Applying Lemma 4.1 yields

$$
\lim _{\varepsilon \searrow 0} \int_{0}^{T}\left|\partial_{t} u^{\varepsilon}(t)\right|_{L^{2}\left(\mathbb{R}^{m}\right)}^{2} \mathrm{~d} t=\int_{0}^{T}\left|\partial_{t} u(t)\right|_{L^{2}\left(\mathbb{R}^{m}\right)}^{2} \mathrm{~d} t
$$

$\lim _{\varepsilon \searrow 0} \int_{0}^{T} \int_{\mathbb{R}^{m}}(D+b \otimes b) \nabla u^{\varepsilon}(t) \cdot \nabla u^{\varepsilon}(t) \mathrm{d} y \mathrm{~d} t=\int_{0}^{T} \int_{\mathbb{R}^{m}}(D+b \otimes b) \nabla u(t) \cdot \nabla u(t) \mathrm{d} y \mathrm{~d} t$ and therefore $\left(\partial_{t} u^{\varepsilon}\right)_{\varepsilon}$ converges strongly toward $\partial_{t} u$ in $L^{2}\left([0, T] ; L^{2}\left(\mathbb{R}^{m}\right)\right)$ and $\left(\nabla u^{\varepsilon}\right)_{\varepsilon}$ converges strongly toward $\nabla u$ in $L^{2}\left([0, T] ; X_{P}\right)$, thanks to the second statement in Proposition 4.1. Coming back in (17) we deduce that (use the hypothesis $\lim _{\varepsilon} \searrow_{0} \varepsilon^{-1} \mid b$. $\left.\left.\nabla u_{\text {in }}^{\varepsilon}\right|_{L^{2}\left(\mathbb{R}^{m}\right)}=0\right)$

$$
\lim _{\varepsilon \searrow 0} \frac{1}{\varepsilon^{2}} \int_{0}^{T}\left|b \cdot \nabla u^{\varepsilon}(t)\right|_{L^{2}\left(\mathbb{R}^{m}\right)}^{2} \mathrm{~d} t=0, \quad T \in \mathbb{R}_{+} .
$$

Notice also that

$$
u^{\varepsilon}(t)-u(t)=u^{\varepsilon}(0)-u(0)+\int_{0}^{t}\left(\partial_{t} u^{\varepsilon}(s)-\partial_{t} u(s)\right) \mathrm{d} s
$$

implying that

$$
\begin{aligned}
\left|u^{\varepsilon}(t)-u(t)\right|_{L^{2}\left(\mathbb{R}^{m}\right)}^{2} & \leq 2\left|u_{\mathrm{in}}^{\varepsilon}-u_{\text {in }}\right|_{L^{2}\left(\mathbb{R}^{m}\right)}^{2}+2 t \int_{0}^{t}\left|\partial_{t} u^{\varepsilon}(s)-\partial_{t} u(s)\right|_{L^{2}\left(\mathbb{R}^{m}\right)}^{2} \mathrm{~d} s \\
& \leq 2\left|u_{\mathrm{in}}^{\varepsilon}-u_{\text {in }}\right|^{2}+2 T\left|\partial_{t} u^{\varepsilon}-\partial_{t} u\right|_{L^{2}\left([0, T] ; L^{2}\left(\mathbb{R}^{m}\right)\right)}^{2}, t \in[0, T] .
\end{aligned}
$$

Therefore $\left(u^{\varepsilon}\right)_{\varepsilon}$ converges strongly toward $u$ in $L_{\text {loc }}^{\infty}\left(\mathbb{R}_{+} ; L^{2}\left(\mathbb{R}^{m}\right)\right)$, as $\varepsilon \searrow 0$.

\section{Remark 4.1}

By the above arguments we know that

$$
\lim _{\varepsilon \searrow 0} \int_{0}^{T} \int_{\mathbb{R}^{m}} D \nabla u^{\varepsilon}(t) \cdot \nabla u^{\varepsilon}(t) \mathrm{d} y \mathrm{~d} t=\int_{0}^{T} \int_{\mathbb{R}^{m}} D \nabla u(t) \cdot \nabla u(t) \mathrm{d} y \mathrm{~d} t
$$

and

$$
\lim _{\varepsilon \searrow 0} \nabla u^{\varepsilon}=\nabla u \text { in } L^{2}\left([0, T] ; X_{P}\right)
$$

As we have

$$
\left|D \nabla u \cdot\left(\nabla u^{\varepsilon}-\nabla u\right)\right|=\left|D\left(\nabla u^{\varepsilon}-\nabla u\right) \cdot \nabla u\right| \leq|D|_{H_{Q}^{\infty}}|\nabla u|_{X_{P}}\left|\nabla u^{\varepsilon}-\nabla u\right|_{X_{P}}
$$

we deduce

$\left|\int_{0}^{T} \int_{\mathbb{R}^{m}} D \nabla u(t) \cdot\left(\nabla u^{\varepsilon}(t)-\nabla u(t)\right) \mathrm{d} y \mathrm{~d} t\right| \leq|D|_{H_{Q}^{\infty}}|\nabla u|_{L^{2}\left([0, T] ; X_{P}\right)}\left|\nabla u^{\varepsilon}-\nabla u\right|_{L^{2}\left([0, T] ; X_{P}\right)} \underset{\varepsilon \searrow 0}{\longrightarrow} 0$.

We obtain

$$
\lim _{\varepsilon \searrow 0} \int_{0}^{T} \int_{\mathbb{R}^{m}} D\left(\nabla u^{\varepsilon}(t)-\nabla u(t)\right) \cdot\left(\nabla u^{\varepsilon}(t)-\nabla u(t)\right) \mathrm{d} y \mathrm{~d} t=0
$$


We also have $\lim _{\varepsilon \searrow 0} \partial_{t} u^{\varepsilon}=\partial_{t} u$ in $L^{2}\left([0, T] ; L^{2}\left(\mathbb{R}^{m}\right)\right)$ and

and therefore

$$
\lim _{\varepsilon \searrow 0} \frac{1}{\varepsilon^{2}} \int_{0}^{T} \int_{\mathbb{R}^{m}}\left(b \cdot \nabla u^{\varepsilon}(t)\right)^{2} \mathrm{~d} y \mathrm{~d} t=0
$$

$$
\lim _{\varepsilon \searrow 0} \int_{0}^{T} \int_{\mathbb{R}^{m}}\left\{\left(\partial_{t} u^{\varepsilon}-\partial_{t} u\right)^{2}+D\left(\nabla u^{\varepsilon}-\nabla u\right) \cdot\left(\nabla u^{\varepsilon}-\nabla u\right)+\frac{\left(b \cdot \nabla u^{\varepsilon}\right)^{2}}{\varepsilon^{2}}\right\} \mathrm{d} y \mathrm{~d} t=0 .
$$

\section{Finite speed propagation}

It is well known that the solution of the wave equation propagates at finite speed. Observe that the spectral radius of the matrix $D+\frac{b \otimes b}{\varepsilon^{2}}$ is of order $\frac{1}{\varepsilon^{2}}$ and therefore the solutions $\left(u^{\varepsilon}\right)_{\varepsilon}$ propagate at speeds of order $\frac{1}{\varepsilon}$, going to infinity as $\varepsilon \searrow 0$. Nevertheless the limit of the family $\left(u^{\varepsilon}\right)_{\varepsilon}$ propagates at finite speed, given by the spectral radius of $\langle D\rangle$ (not depending on $\varepsilon$ ). More exactly, in the case of the limit model, we have the following result.

\section{Proposition 5.1}

Under the hypotheses of Proposition 3.4, let us consider the unique variational solution of (11), (12) corresponding to the initial conditions $\left(u_{\text {in }}, \dot{u}_{\text {in }}\right) \in H_{P}^{1} \times \operatorname{dom} \mathcal{T}$. Let $c>0$ be a real number such that

$$
\langle D\rangle(y) \leq c^{2} I_{m}, \quad y \in \mathbb{R}^{m} .
$$

Assume that for some $R>0$, the initial conditions satisfy

$$
u_{\text {in }}(y)=0, \quad \dot{u}_{\text {in }}(y)=0, \quad|y|>R .
$$

Then we have

$$
u(t, y)=0, \quad \partial_{t} u(t, y)=0, \quad|y|>R+c t .
$$

Proof. Pick a non-decreasing function $\theta \in C_{b}^{1}(\mathbb{R})$ such that $\theta(r)=0$ if $r \leq 0, \theta(r)>0$ if $r>0$. Using the variational formulation with the function

$$
(t, y) \rightarrow \theta\left(\sqrt{|y|^{2}+\delta^{2}}-R-c t\right) \partial_{t} u, \quad \delta>0
$$

yields

$$
\begin{aligned}
& \frac{1}{2} \frac{\mathrm{d}}{\mathrm{d} t} \int_{\mathbb{R}^{m}}\left(\partial_{t} u\right)^{2} \theta\left(\sqrt{|y|^{2}+\delta^{2}}-R-c t\right) \mathrm{d} y+\frac{c}{2} \int_{\mathbb{R}^{m}}\left[\left(\partial_{t} u\right)^{2}+\langle D\rangle \nabla u \cdot \nabla u\right] \theta^{\prime} \mathrm{d} y \\
& +\frac{1}{2} \frac{\mathrm{d}}{\mathrm{d} t} \int_{\mathbb{R}^{m}}\langle D\rangle \nabla u \cdot \nabla u \theta\left(\sqrt{|y|^{2}+\delta^{2}}-R-c t\right) \mathrm{d} y+\int_{\mathbb{R}^{m}}\langle D\rangle \nabla u \cdot \frac{y}{\sqrt{|y|^{2}+\delta^{2}}} \partial_{t} u \theta^{\prime} \mathrm{d} y=0 .
\end{aligned}
$$

Notice that we have for any $(t, y) \in \mathbb{R}_{+} \times \mathbb{R}^{m}$

$$
\begin{aligned}
2\left|\langle D\rangle \nabla u \cdot \frac{y}{\sqrt{|y|^{2}+\delta^{2}}} \partial_{t} u\right| & \leq c\left(\partial_{t} u\right)^{2}+\frac{1}{c}\left(\langle D\rangle \nabla u \cdot \frac{y}{\sqrt{|y|^{2}+\delta^{2}}}\right)^{2} \\
& \leq c\left(\partial_{t} u\right)^{2}+\frac{1}{c}(\langle D\rangle \nabla u \cdot \nabla u)\left(\langle D\rangle \frac{y}{\sqrt{|y|^{2}+\delta^{2}}} \cdot \frac{y}{\sqrt{|y|^{2}+\delta^{2}}}\right) \\
& \leq c\left(\partial_{t} u\right)^{2}+\frac{\langle D\rangle \nabla u \cdot \nabla u}{c} \frac{c^{2}|y|^{2}}{|y|^{2}+\delta^{2}} \\
& \leq c\left[\left(\partial_{t} u\right)^{2}+\langle D\rangle \nabla u \cdot \nabla u\right] .
\end{aligned}
$$


As $\theta^{\prime} \geq 0$, we deduce

$$
\frac{\mathrm{d}}{\mathrm{d} t} \int_{\mathbb{R}^{m}}\left[\left(\partial_{t} u\right)^{2}+\langle D\rangle \nabla u \cdot \nabla u\right] \theta\left(\sqrt{|y|^{2}+\delta^{2}}-R-c t\right) \mathrm{d} y \leq 0
$$

implying that

$$
\begin{aligned}
& \int_{\mathbb{R}^{m}}\left[\left(\partial_{t} u(t, y)\right)^{2}+\langle D\rangle \nabla u(t, y) \cdot \nabla u(t, y)\right] \theta\left(\sqrt{|y|^{2}+\delta^{2}}-R-c t\right) \mathrm{d} y \\
& \leq \int_{\mathbb{R}^{m}}\left[\left(\dot{u}_{\text {in }}\right)^{2}+\langle D\rangle \nabla u_{\text {in }} \cdot \nabla u_{\text {in }}\right] \theta\left(\sqrt{|y|^{2}+\delta^{2}}-R\right) \mathrm{d} y .
\end{aligned}
$$

As $\theta$ is non-decreasing, we have

$$
\begin{aligned}
& \int_{\mathbb{R}^{m}}\left[\left(\partial_{t} u(t, y)\right)^{2}+\langle D\rangle \nabla u(t, y) \cdot \nabla u(t, y)\right] \theta(|y|-R-c t) \mathrm{d} y \\
& \leq \int_{\mathbb{R}^{m}}\left[\left(\partial_{t} u(t, y)\right)^{2}+\langle D\rangle \nabla u(t, y) \cdot \nabla u(t, y)\right] \theta\left(\sqrt{|y|^{2}+\delta^{2}}-R-c t\right) \mathrm{d} y
\end{aligned}
$$

and thus

$$
\begin{aligned}
& \int_{\mathbb{R}^{m}}\left[\left(\partial_{t} u(t, y)\right)^{2}+\langle D\rangle \nabla u(t, y) \cdot \nabla u(t, y)\right] \theta(|y|-R-c t) \mathrm{d} y \\
& \leq \int_{\mathbb{R}^{m}}\left[\left(\dot{u}_{\text {in }}\right)^{2}+\langle D\rangle \nabla u_{\text {in }} \cdot \nabla u_{\text {in }}\right] \theta\left(\sqrt{|y|^{2}+\delta^{2}}-R\right) \mathrm{d} y .
\end{aligned}
$$

By dominated convergence, letting $\delta \searrow 0$, we obtain

$$
\begin{aligned}
& \int_{\mathbb{R}^{m}}\left[\left(\partial_{t} u(t, y)\right)^{2}+\langle D\rangle \nabla u(t, y) \cdot \nabla u(t, y)\right] \theta(|y|-R-c t) \mathrm{d} y \\
& \leq \int_{\mathbb{R}^{m}}\left[\left(\dot{u}_{\text {in }}\right)^{2}+\langle D\rangle \nabla u_{\text {in }} \cdot \nabla u_{\text {in }}\right] \theta(|y|-R) \mathrm{d} y=0
\end{aligned}
$$

implying that

$$
\partial_{t} u(t, y)=0, \quad \nabla u(t, y)=0, \quad|y|>R+c t .
$$

Moreover, for any $(t, y) \in \mathbb{R}_{+} \times \mathbb{R}^{m}$ such that $|y|>R+c t$, we have

$$
u(t, y)=u_{\text {in }}(y)+\int_{0}^{t} \partial_{t} u(s, y) \mathrm{d} s=u_{\text {in }}(y)=0
$$

since $|y|>R+c t \geq R+c s \geq R$ for any $s \in[0, T]$.

\section{Remark 5.1}

Under the hypotheses of Theorem 4.2 we can show that the energy of the solutions $\left(u^{\varepsilon}\right)_{\varepsilon}$ outside the propagation cone of the limit solution $u$ is negligible. Indeed, by Remark 4.1, using the notations in Proposition 5.1 we write

$$
\begin{aligned}
& \int_{0}^{T} \int_{|y| \leq R+c t}\left\{\left(\partial_{t} u^{\varepsilon}-\partial_{t} u\right)^{2}+D\left(\nabla u^{\varepsilon}-\nabla u\right) \cdot\left(\nabla u^{\varepsilon}-\nabla u\right)+\frac{\left(b \cdot \nabla u^{\varepsilon}\right)^{2}}{\varepsilon^{2}}\right\} \mathrm{d} y \mathrm{~d} t \\
& +\int_{0}^{T} \int_{|y|>R+c t}\left\{\left(\partial_{t} u^{\varepsilon}\right)^{2}+D \nabla u^{\varepsilon} \cdot \nabla u^{\varepsilon}+\frac{\left(b \cdot \nabla u^{\varepsilon}\right)^{2}}{\varepsilon^{2}}\right\} \mathrm{d} y \mathrm{~d} t=o(1) \text { as } \varepsilon \searrow 0
\end{aligned}
$$

which implies that

$$
\int_{0}^{T} \int_{|y|>R+c t}\left\{\left(\partial_{t} u^{\varepsilon}\right)^{2}+D \nabla u^{\varepsilon} \cdot \nabla u^{\varepsilon}+\frac{\left(b \cdot \nabla u^{\varepsilon}\right)^{2}}{\varepsilon^{2}}\right\} \mathrm{d} y \mathrm{~d} t=o(1) \text { as } \varepsilon \searrow 0 .
$$


It may happen that an explicit expression for the average matrix field $\langle D\rangle$ is not available, and therefore we can not compute the spectral radius of $\langle D\rangle$. Nevertheless we can estimate the propagation speed of the solution for the limit model in terms of the spectrum of $D$.

\section{Proposition 5.2}

Let us consider

$$
\Lambda(y):=\sup _{\xi \in \mathbb{R}^{m} \backslash\{0\}} \frac{D(y) \xi \cdot \xi}{|\xi|^{2}}, \quad c^{2}(y):=\sup _{\xi \in \mathbb{R}^{m} \backslash\{0\}} \frac{\langle D\rangle(y) \xi \cdot \xi}{|\xi|^{2}}, y \in \mathbb{R}^{m} .
$$

Then we have the inequality

$$
c(y) \leq\langle\Lambda\rangle^{1 / 2}(y)\left|Q^{1 / 2}\right|_{L^{\infty}}\left|P^{1 / 2}\right|_{L^{\infty}}, \quad y \in \mathbb{R}^{m} .
$$

In particular, if $\Lambda \in L^{\infty}\left(\mathbb{R}^{m}\right)$, then $c \in L^{\infty}\left(\mathbb{R}^{m}\right)$ and

$$
|c|_{L^{\infty}} \leq|\Lambda|_{L^{\infty}}^{1 / 2}\left|Q^{1 / 2}\right|_{L^{\infty}}\left|P^{1 / 2}\right|_{L^{\infty}}
$$

Proof. By the hypothesis, we have for any $y \in \mathbb{R}^{m}, \xi \in \mathbb{R}^{m}$

$$
Q^{1 / 2}(y) D(y) Q^{1 / 2}(y): \xi \otimes \xi \leq \Lambda(y)\left|Q^{1 / 2}(y) \xi\right|^{2} \leq \Lambda(y)\left|Q^{1 / 2}(y)\right|^{2}|\xi|^{2} .
$$

As in the proof of Proposition 2.3, we write

$$
\begin{aligned}
Q^{1 / 2} G(s) D Q^{1 / 2}: \xi \otimes \xi & ={ }^{t} \mathcal{O}(s ; y) Q_{s}^{1 / 2} D_{s} Q_{s}^{1 / 2} \mathcal{O}(s ; y): \xi \otimes \xi \\
& =\left(Q^{1 / 2} D Q^{1 / 2}\right)_{s}: \mathcal{O}(s ; y) \xi \otimes \mathcal{O}(s ; y) \xi \\
& \leq \Lambda(Y(s ; y))\left|Q_{s}^{1 / 2}\right|^{2}|\xi|^{2} \\
& \leq \Lambda(Y(s ; y))\left|Q^{1 / 2}\right|_{L^{\infty}}^{2}|\xi|^{2}
\end{aligned}
$$

implying that

$$
Q^{1 / 2}\langle D\rangle Q^{1 / 2}: \xi \otimes \xi \leq\langle\Lambda\rangle\left|Q^{1 / 2}\right|_{L^{\infty}}^{2}|\xi|^{2} .
$$

Replacing $\xi$ by $P^{1 / 2} \xi$ yields

$$
\langle D\rangle: \xi \otimes \xi \leq\langle\Lambda\rangle\left|Q^{1 / 2}\right|_{L^{\infty}}^{2}\left|P^{1 / 2}\right|_{L^{\infty}}^{2}|\xi|^{2} .
$$

Therefore we obtain

$$
c^{2}(y)=\sup _{\xi \in \mathbb{R}^{m} \backslash\{0\}} \frac{\langle D\rangle(y) \xi \cdot \xi}{|\xi|^{2}} \leq\langle\Lambda\rangle(y)\left|Q^{1 / 2}\right|_{L^{\infty}}^{2}\left|P^{1 / 2}\right|_{L^{\infty}}^{2}
$$

and thus

$$
c(y) \leq\langle\Lambda\rangle^{1 / 2}(y)\left|Q^{1 / 2}\right|_{L^{\infty}}\left|P^{1 / 2}\right|_{L^{\infty}} .
$$




\section{A Proofs of Proposition 2.3, Theorem 2.2, Propo- sition 2.5, Lemma 2.1}

We indicate here some proof details concerning the properties of the average operators for matrix fields and functions.

Proof. (of Proposition 2.3)

1. By the characterization in Proposition 2.2 we know that

$$
P_{s}=\partial Y(s ; \cdot) P^{t} \partial Y(s ; \cdot), \quad s \in \mathbb{R} .
$$

For any $s \in \mathbb{R}$ we consider the matrix field $\mathcal{O}(s ; \cdot)=Q_{s}^{1 / 2} \partial Y(s ; \cdot) Q^{-1 / 2}$. Observe that $\mathcal{O}(s ; \cdot)$ is a field of orthogonal matrices, for any $s \in \mathbb{R}$. Indeed we have, thanks to (18)

$$
\begin{aligned}
{ }^{t} \mathcal{O}(s ; \cdot) \mathcal{O}(s ; \cdot) & =Q^{-1 / 2}{ }^{t} \partial Y(s ; \cdot) Q_{s}^{1 / 2} Q_{s}^{1 / 2} \partial Y(s ; \cdot) Q^{-1 / 2} \\
& =Q^{-1 / 2}\left(\partial Y^{-1}(s ; \cdot) P_{s}{ }^{t} \partial Y^{-1}(s ; \cdot)\right)^{-1} Q^{-1 / 2} \\
& =Q^{-1 / 2} P^{-1} Q^{-1 / 2} \\
& =I_{m}
\end{aligned}
$$

implying that for any matrix field $A$ we have

$$
Q^{1 / 2} G(s) A Q^{1 / 2}=Q^{1 / 2} \partial Y^{-1}(s ; \cdot) A_{s}{ }^{t} \partial Y^{-1}(s ; \cdot) Q^{1 / 2}={ }^{t} \mathcal{O}(s ; \cdot) Q_{s}^{1 / 2} A_{s} Q_{s}^{1 / 2} \mathcal{O}(s ; \cdot) .
$$

It is easily seen that if $A \in H_{Q}$, then for any $s \in \mathbb{R}$

$$
\begin{aligned}
|G(s) A|_{Q}^{2} & =\int_{\mathbb{R}^{m}} Q^{1 / 2} G(s) A Q^{1 / 2}: Q^{1 / 2} G(s) A Q^{1 / 2} \mathrm{~d} y \\
& =\int_{\mathbb{R}^{m}}{ }^{t} \mathcal{O}(s ; \cdot) Q_{s}^{1 / 2} A_{s} Q_{s}^{1 / 2} \mathcal{O}(s ; \cdot):{ }^{t} \mathcal{O}(s ; \cdot) Q_{s}^{1 / 2} A_{s} Q_{s}^{1 / 2} \mathcal{O}(s ; \cdot) \mathrm{d} y \\
& =\int_{\mathbb{R}^{m}} Q_{s}^{1 / 2} A_{s} Q_{s}^{1 / 2}: Q_{s}^{1 / 2} A_{s} Q_{s}^{1 / 2} \mathrm{~d} y \\
& =\int_{\mathbb{R}^{m}} Q^{1 / 2} A Q^{1 / 2}: Q^{1 / 2} A Q^{1 / 2} \mathrm{~d} y=|A|_{H_{Q}}^{2}
\end{aligned}
$$

proving that $G(s)$ is a unitary transformation for any $s \in \mathbb{R}$. The group property of the family $(G(s))_{s \in \mathbb{R}}$ follows easily from the group property of the flow $(Y(s ; \cdot))_{s \in \mathbb{R}}$

$$
\begin{aligned}
G(s) G(t) A & =\partial Y^{-1}(s ; \cdot)(G(t) A)_{s}{ }^{t} \partial Y^{-1}(s ; \cdot) \\
& =\partial Y^{-1}(s ; \cdot) \partial Y^{-1}(t ; Y(s ; \cdot))\left(A_{t}\right)_{s}{ }^{t} \partial Y^{-1}(t ; Y(s ; \cdot))^{t} \partial Y^{-1}(s ; \cdot) \\
& =\partial Y^{-1}(t+s ; \cdot) A_{t+s}{ }^{t} \partial Y^{-1}(t+s ; \cdot)=G(t+s) A, \quad A \in H_{Q} .
\end{aligned}
$$

The continuity of the group, i.e., $\lim _{s \rightarrow 0} G(s) A=A$ strongly in $H_{Q}$, comes by standard arguments.

2. Notice that $G(s)$ commutes with transposition

$$
\begin{aligned}
{ }^{t}(G(s) A) & ={ }^{t}\left(\partial Y^{-1}(s ; \cdot) A_{s}{ }^{t} \partial Y^{-1}(s ; \cdot)\right) \\
& =\partial Y^{-1}(s ; \cdot){ }^{t} A_{s}{ }^{t} \partial Y^{-1}(s ; \cdot) \\
& =G(s){ }^{t} A .
\end{aligned}
$$


In particular, if ${ }^{t} A=A$, then ${ }^{t}(G(s) A)=G(s) A$.

3. We use the formula (19). For any $\xi \in \mathbb{R}^{m}$ we have

$$
\begin{aligned}
G(s) A: Q^{1 / 2} \xi \otimes Q^{1 / 2} \xi & =Q^{1 / 2} G(s) A Q^{1 / 2}: \xi \otimes \xi \\
& ={ }^{t} \mathcal{O}(s ; \cdot) Q_{s}^{1 / 2} A_{s} Q_{s}^{1 / 2} \mathcal{O}(s ; \cdot): \xi \otimes \xi \\
& =Q_{s}^{1 / 2} A_{s} Q_{s}^{1 / 2}: \mathcal{O}(s ; \cdot)(\xi \otimes \xi){ }^{t} \mathcal{O}(s ; \cdot) \\
& =Q_{s}^{1 / 2} A_{s} Q_{s}^{1 / 2}:(\mathcal{O}(s ; \cdot) \xi) \otimes(\mathcal{O}(s ; \cdot) \xi) \\
& =A_{s}:\left(Q_{s}^{1 / 2} \mathcal{O}(s ; \cdot) \xi\right) \otimes\left(Q_{s}^{1 / 2} \mathcal{O}(s ; \cdot) \xi\right) .
\end{aligned}
$$

As $A$ is a field of positive semi-definite matrices, therefore $G(s) A$ is a field of positive semi-definite matrices as well.

4. Assume that there is $\alpha>0$ such that $Q^{1 / 2} A Q^{1 / 2} \geq \alpha I_{m}$ on $\mathcal{S}$. As before we write for any $\xi \in \mathbb{R}^{m}, y \in \mathcal{S}$

$$
Q^{1 / 2} G(s) A Q^{1 / 2}: \xi \otimes \xi=\left(Q^{1 / 2} A Q^{1 / 2}\right)_{s}:(\mathcal{O}(s ; \cdot) \xi) \otimes(\mathcal{O}(s ; \cdot) \xi) \geq \alpha|\mathcal{O}(s ; \cdot) \xi|^{2}=\alpha|\xi|^{2}
$$

saying that $Q^{1 / 2} G(s) A Q^{1 / 2} \geq \alpha I_{m}$ on $\mathcal{S}$.

5. Here $G(s)$ stands for the application $A \rightarrow \partial Y(-s ; Y(s ; \cdot)) A(Y(s ; \cdot))^{t} \partial Y(-s ; Y(s ; \cdot))$ independently of $A$ being in $H_{Q}$ or in $H_{Q \text {,loc }}$. As $\psi$ is left invariant by the flow of $b$, so is $\mathbf{1}_{\{\psi \leq k\}}$, for any $k \in \mathbb{N}$. If $A$ belongs to $H_{Q \text {,loc }}$, we have

$$
\mathbf{1}_{\{\psi \leq k\}} G(s) A=G(s)\left(\mathbf{1}_{\{\psi \leq k\}} A\right) \in H_{Q}, \quad k \in \mathbb{N}, \quad s \in \mathbb{R}
$$

saying that $G(s) A \in H_{Q \text {,loc }}, s \in \mathbb{R}$. Moreover, the applications $(G(s))_{s \in \mathbb{R}}$ preserve locally the norm of $H_{Q}$

$$
\left|\mathbf{1}_{\{\psi \leq k\}} G(s) A\right|_{H_{Q}}=\left|G(s)\left(\mathbf{1}_{\{\psi \leq k\}} A\right)\right|_{H_{Q}}=\left|\mathbf{1}_{\{\psi \leq k\}} A\right|_{H_{Q}}, \quad k \in \mathbb{N}, \quad s \in \mathbb{R} .
$$

Proof. (of Theorem 2.2)

The first and second statements are immediate.

3. For any $\xi \in \mathbb{R}^{m}, \chi \in C_{c}^{0}(\mathcal{S}), \chi \geq 0$ we have $\chi(\cdot) P^{1 / 2} \xi \otimes P^{1 / 2} \xi \in H_{Q}$ and we can write, thanks to (19)

$$
\begin{aligned}
\left(G(s) A, \chi(\cdot) P^{1 / 2} \xi \otimes P^{1 / 2} \xi\right)_{Q} & =\int_{\mathbb{R}^{m}} \chi(y) Q^{1 / 2} G(s) A Q^{1 / 2}: \xi \otimes \xi \mathrm{d} y \\
& =\int_{\mathbb{R}^{m}} \chi(y){ }^{t} \mathcal{O}(s ; y) Q_{s}^{1 / 2} A_{s} Q_{s}^{1 / 2} \mathcal{O}(s ; y) \xi \cdot \xi \mathrm{d} y \\
& =\int_{\mathbb{R}^{m}} \chi(y) Q_{s}^{1 / 2} A_{s} Q_{s}^{1 / 2}: \mathcal{O}(s ; y) \xi \otimes \mathcal{O}(s ; y) \xi \mathrm{d} y \\
& \geq \alpha \int_{\mathbb{R}^{m}}|\mathcal{O}(s ; y) \xi|^{2} \chi(y) \mathrm{d} y \\
& =\alpha|\xi|^{2} \int_{\mathbb{R}^{m}} \chi(y) \mathrm{d} y .
\end{aligned}
$$

Taking the average over $[0, S]$ and letting $S \rightarrow+\infty$ yield

$$
\int_{\mathbb{R}^{m}} \chi(y) Q^{1 / 2}\langle A\rangle Q^{1 / 2}: \xi \otimes \xi \mathrm{d} y=\left(\langle A\rangle, \chi P^{1 / 2} \xi \otimes P^{1 / 2} \xi\right)_{Q} \geq \int_{\mathbb{R}^{m}} \alpha|\xi|^{2} \chi(y) \mathrm{d} y
$$


implying that

$$
Q^{1 / 2}(y)\langle A\rangle(y) Q^{1 / 2}(y) \geq \alpha I_{m}, \quad y \in \mathcal{S} .
$$

4. For any $A \in H_{Q}$, we have by the properties of the orthogonal projection on ker $L$ that $|\langle A\rangle|_{H_{Q}}=\left|\operatorname{Proj}_{\text {ker } L} A\right|_{H_{Q}} \leq|A|_{H_{Q}}$. For the last inequality, consider $M \in \mathcal{M}_{m}(\mathbb{R})$ a fixed matrix, $\chi \in C_{c}^{0}\left(\mathbb{R}^{m}\right), \chi \geq 0$ and, as before, observe that $\chi P^{1 / 2} M P^{1 / 2} \in H_{Q}$, which allows us to write

$$
\begin{aligned}
(G(s) A, & \left.\chi P^{1 / 2} M P^{1 / 2}\right)_{Q}=\int_{\mathbb{R}^{m}} Q^{1 / 2} G(s) A Q^{1 / 2}: \chi M \mathrm{~d} y \\
& =\int_{\mathbb{R}^{m}}{ }^{t} \mathcal{O}(s ; y) Q_{s}^{1 / 2} A_{s} Q_{s}^{1 / 2} \mathcal{O}(s ; y): \chi M \mathrm{~d} y \\
& =\int_{\mathbb{R}^{m}} Q_{s}^{1 / 2} A_{s} Q_{s}^{1 / 2}: \mathcal{O}(s ; y) M^{t} \mathcal{O}(s ; y) \chi \mathrm{d} y \\
& \leq \int_{\mathbb{R}^{m}} \sqrt{Q_{s}^{1 / 2} A_{s} Q_{s}^{1 / 2}: Q_{s}^{1 / 2} A_{s} Q_{s}^{1 / 2}} \sqrt{\mathcal{O}(s ; y) M^{t} \mathcal{O}(s ; y): \mathcal{O}(s ; y) M^{t} \mathcal{O}(s ; y)} \chi \mathrm{d} y \\
& \leq|A|_{H_{Q}^{\infty}}(M: M)^{1 / 2} \int_{\mathbb{R}^{m}} \chi(y) \mathrm{d} y .
\end{aligned}
$$

Taking the average over $[0, S]$ and letting $S \rightarrow+\infty$, lead to

$$
\int_{\mathbb{R}^{m}} Q^{1 / 2}\langle A\rangle Q^{1 / 2}: M \chi(y) \mathrm{d} y=\left(\langle A\rangle, \chi P^{1 / 2} M P^{1 / 2}\right)_{Q} \leq|A|_{H_{Q}^{\infty}}(M: M)^{1 / 2} \int_{\mathbb{R}^{m}} \chi(y) \mathrm{d} y .
$$

We deduce that

$$
Q^{1 / 2}(y)\langle A\rangle(y) Q^{1 / 2}(y): M \leq|A|_{H_{Q}^{\infty}}(M: M)^{1 / 2}, \quad y \in \mathbb{R}^{m}, \quad M \in \mathcal{M}_{m}(\mathbb{R})
$$

saying that

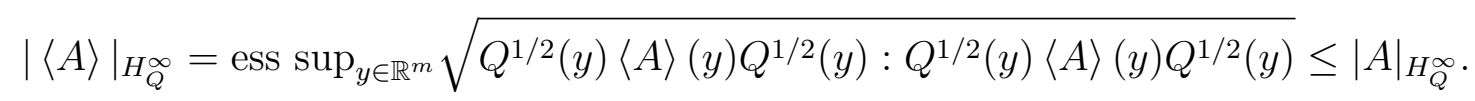

5. Let $A$ be a matrix field in $H_{Q, \text { loc }}$. For any $k \in \mathbb{N}, \mathbf{1}_{\{\psi \leq k\}} A$ belongs to $H_{Q}$, and by the first statement we know that

$$
\lim _{S \rightarrow+\infty} \frac{1}{S} \int_{r}^{r+S} G(s)\left(\mathbf{1}_{\{\psi \leq k\}} A\right) \mathrm{d} s=\left\langle\mathbf{1}_{\{\psi \leq k\}} A\right\rangle \in H_{Q}
$$

uniformly with respect to $r \in \mathbb{R}$, for any fixed $k \in \mathbb{N}$. Observe that for any $k, l \in \mathbb{N}$ we have

$$
\lim _{S \rightarrow+\infty} \frac{1}{S} \int_{0}^{S} G(s)\left(\mathbf{1}_{\{\psi \leq k\}} A\right) \mathrm{d} s=\lim _{S \rightarrow+\infty} \frac{1}{S} \int_{0}^{S} G(s)\left(\mathbf{1}_{\{\psi \leq l\}} A\right) \mathrm{d} s
$$

almost everywhere on $\{\psi \leq \min (k, l)\}$, and thus, there is a matrix field denoted by $\langle A\rangle$, whose restriction on $\{\psi \leq k\}$ coincides with $\left\langle\mathbf{1}_{\{\psi \leq k\}} A\right\rangle$ for any $k \in \mathbb{N}$. Notice also that for any $k \in \mathbb{N}$ we have $\left\langle\mathbf{1}_{\{\psi \leq k\}} A\right\rangle=0$ almost everywhere on $\{\psi>k\}$ and thus we obtain

$$
\mathbf{1}_{\{\psi \leq k\}}\langle A\rangle=\left\langle\mathbf{1}_{\{\psi \leq k\}} A\right\rangle, \quad k \in \mathbb{N} .
$$

Observe that for any $k \in \mathbb{N}$, we have the uniform, with respect to $r \in \mathbb{R}$, convergence in $H_{Q}$

$$
\lim _{S \rightarrow+\infty} \mathbf{1}_{\{\psi \leq k\}} \frac{1}{S} \int_{r}^{r+S} G(s)(A) \mathrm{d} s=\lim _{S \rightarrow+\infty} \frac{1}{S} \int_{r}^{r+S} G(s)\left(\mathbf{1}_{\{\psi \leq k\}} A\right) \mathrm{d} s=\left\langle\mathbf{1}_{\{\psi \leq k\}} A\right\rangle=\mathbf{1}_{\{\psi \leq k\}}\langle A\rangle
$$


saying that $\lim _{S \rightarrow+\infty} \frac{1}{S} \int_{r}^{r+S} G(s) A \mathrm{~d} s=\langle A\rangle$ in $H_{Q \text {,loc }}$ (uniformly with respect to $r \in \mathbb{R}$,

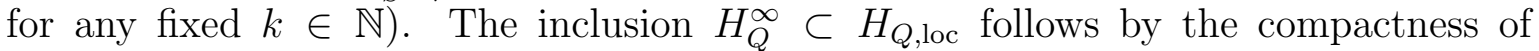
$\{\psi \leq k\}, k \in \mathbb{N}$. By the fourth statement we have

$$
|\langle A\rangle|_{H_{Q}^{\infty}}=\sup _{k \in \mathbb{N}}\left|\mathbf{1}_{\{\psi \leq k\}}\langle A\rangle\right|_{H_{Q}^{\infty}}=\sup _{k \in \mathbb{N}}\left|\left\langle\mathbf{1}_{\{\psi \leq k\}} A\right\rangle\right|_{H_{Q}^{\infty}} \leq \sup _{k \in \mathbb{N}}\left|\mathbf{1}_{\{\psi \leq k\}} A\right|_{H_{Q}^{\infty}}=|A|_{H_{Q}^{\infty}} .
$$

Let $A$ be a matrix field of $H_{Q \text {,loc }}$, such that $Q^{1 / 2}(y) A(y) Q^{1 / 2}(y) \geq \alpha I_{m}, y \in \mathbb{R}^{m}$, for some $\alpha>0$. For any $k \in \mathbb{N}$ we have $\mathbf{1}_{\{\psi \leq k\}} A \in H_{Q}$ and

$$
Q^{1 / 2}(y) \mathbf{1}_{\{\psi \leq k\}} A(y) Q^{1 / 2}(y) \geq \alpha I_{m}, \quad y \in\{\psi \leq k\} .
$$

By the third statement we deduce that for any $k \in \mathbb{N}$

$$
Q^{1 / 2}(y) \mathbf{1}_{\{\psi \leq k\}}\langle A\rangle(y) Q^{1 / 2}(y)=Q^{1 / 2}(y)\left\langle\mathbf{1}_{\{\psi \leq k\}} A\right\rangle(y) Q^{1 / 2}(y) \geq \alpha I_{m}, \quad y \in\{\psi \leq k\}
$$

saying that $Q^{1 / 2}(y)\langle A\rangle(y) Q^{1 / 2}(y) \geq \alpha I_{m}, \quad y \in \mathbb{R}^{m}$.

Proof. (of Proposition 2.5)

Let $u=u(y)$ be a function in $H_{P}^{1}$. As the flow satisfies $Y \in W_{\text {loc }}^{1, \infty}\left(\mathbb{R} \times \mathbb{R}^{m}\right)$, we have $\nabla u_{s}={ }^{t} \partial Y(s ; \cdot)(\nabla u)_{s}$. By Proposition 2.2 we know that $P_{s}=\partial Y(s ; \cdot) P^{t} \partial Y(s ; \cdot)$, and therefore we can write

$$
\begin{aligned}
\left|u_{s}\right|_{H_{P}^{1}}^{2} & =\int_{\mathbb{R}^{m}}\left(u_{s}(y)\right)^{2} \mathrm{~d} y+\int_{\mathbb{R}^{m}} P(y) \nabla u_{s} \cdot \nabla u_{s} \mathrm{~d} y \\
& =\int_{\mathbb{R}^{m}}(u(y))^{2} \mathrm{~d} y+\int_{\mathbb{R}^{m}} \underbrace{\partial Y(s ; y) P(y)^{t} \partial Y(s ; y)}_{P_{s}}:(\nabla u)_{s} \otimes(\nabla u)_{s} \mathrm{~d} y \\
& =|u|_{L^{2}\left(\mathbb{R}^{m}\right)}^{2}+|\nabla u|_{X_{P}}^{2}=|u|_{H_{P}^{1}}^{2} .
\end{aligned}
$$

The group property of $\left(\zeta^{1}(s)\right)_{s \in \mathbb{R}}$ comes by the group property of $(\zeta(s))_{s \in \mathbb{R}}$. In order to check the continuity of $\left(\zeta^{1}(s)\right)_{s \in \mathbb{R}}$, observe that for any $u \in H_{P}^{1}$, we have

$$
\begin{aligned}
\left|\zeta^{1}(s) u-u\right|_{H_{P}^{1}}^{2} & -|\zeta(s) u-u|_{L^{2}\left(\mathbb{R}^{m}\right)}^{2}=\left|\nabla u_{s}-\nabla u\right|_{X_{P}}^{2} \\
& =2|\nabla u|_{X_{P}}^{2}-2\left(\nabla u_{s}, \nabla u\right)_{X_{P}} \\
& =2|\nabla u|_{X_{P}}^{2}-2 \int_{\mathbb{R}^{m}} \underbrace{P^{1 / 2}(y)^{t} \partial Y(s ; y)}_{{ }^{t} \mathcal{O}(s ; y) P_{s}^{1 / 2}}(\nabla u)_{s} \cdot P^{1 / 2}(y) \nabla u \mathrm{~d} y \\
& =2|\nabla u|_{X_{P}}^{2}-2 \int_{\mathbb{R}^{m}}{ }^{t} \mathcal{O}(s ; y)\left(P^{1 / 2} \nabla u\right)_{s} \cdot P^{1 / 2} \nabla u \mathrm{~d} y \\
& =\left|\left(P^{1 / 2} \nabla u\right)_{s}-P^{1 / 2} \nabla u\right|_{L^{2}\left(\mathbb{R}^{m}\right)}^{2}-2 \int_{\mathbb{R}^{m}}\left(P^{1 / 2} \nabla u\right)_{s} \cdot\left(\mathcal{O}-I_{m}\right) P^{1 / 2} \nabla u \mathrm{~d} y .
\end{aligned}
$$

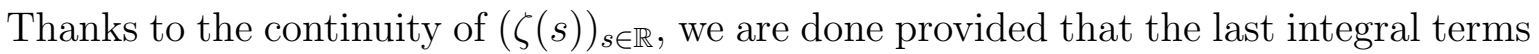
converges to 0 , as $s \rightarrow 0$. The convergence $\lim _{s \rightarrow 0} \partial Y(s ; y)=I_{m}, y \in \mathbb{R}^{m}$, implies the convergences

$$
\begin{gathered}
\lim _{s \rightarrow 0} P(Y(s ; y))=\lim _{s \rightarrow 0} \partial Y(s ; y) P(y)^{t} \partial Y(s ; y)=P(y), \lim _{s \rightarrow 0} P^{1 / 2}(Y(s ; y))=P^{1 / 2}(y) \\
\lim _{s \rightarrow 0} Q(Y(s ; y))=\lim _{s \rightarrow 0}{ }^{t} \partial Y^{-1}(s ; y) Q(y) \partial Y^{-1}(s ; y)=Q(y), \lim _{s \rightarrow 0} Q^{1 / 2}(Y(s ; y))=Q^{1 / 2}(y)
\end{gathered}
$$




$$
\lim _{s \rightarrow 0} \mathcal{O}(s ; y)=\lim _{s \rightarrow 0} Q^{1 / 2}(Y(s ; y)) \partial Y(s ; y) Q^{-1 / 2}(y)=I_{m}, \quad y \in \mathbb{R}^{m} .
$$

Since the matrix $\mathcal{O}(s ; y)$ is orthogonal, we have $|\mathcal{O}(s ; y)|=1$ for any $s \in \mathbb{R}, y \in \mathbb{R}^{m}$, and by the dominated convergence theorem we obtain

$$
\lim _{s \rightarrow 0} \int_{\mathbb{R}^{m}} P^{1 / 2} \nabla u \cdot\left(\mathcal{O}(s ; y)-I_{m}\right) P^{1 / 2} \nabla u \mathrm{~d} y=0
$$

implying that

$$
\begin{aligned}
& \lim _{s \rightarrow 0} \int_{\mathbb{R}^{m}}\left(P^{1 / 2} \nabla u\right)_{s} \cdot\left(\mathcal{O}(s ; y)-I_{m}\right) P^{1 / 2} \nabla u \mathrm{~d} y \\
& =\lim _{s \rightarrow 0} \int_{\mathbb{R}^{m}}\left[\left(P^{1 / 2} \nabla u\right)_{s}-P^{1 / 2} \nabla u\right] \cdot\left(\mathcal{O}(s ; y)-I_{m}\right) P^{1 / 2} \nabla u \mathrm{~d} y=0 .
\end{aligned}
$$

In the last limit we have used the convergence $\lim _{s \rightarrow 0}\left(P^{1 / 2} \nabla u\right)_{s}=P^{1 / 2} \nabla u$ in $L^{2}\left(\mathbb{R}^{m}\right)$, and the upper bound $\left|\mathcal{O}(s ; y)-I_{m}\right| \leq 2, s \in \mathbb{R}, y \in \mathbb{R}^{m}$. By Theorem 2.1 we deduce the strong convergence in $H_{P}^{1}$

$$
\lim _{S \rightarrow+\infty} \frac{1}{S} \int_{r}^{r+S} \zeta^{1}(s) u \mathrm{~d} s=\operatorname{Proj}_{\mathrm{ker} \mathcal{T} \cap H_{P}^{1}} u
$$

implying that $\langle u\rangle=\operatorname{Proj}_{\text {ker } \mathcal{T}} u=\operatorname{Proj}_{\operatorname{ker} \mathcal{T} \cap H_{P}^{1}} u \in H_{P}^{1},(\nabla u-\nabla\langle u\rangle, \nabla v)_{X_{P}}=0$ for any $v \in \operatorname{ker} \mathcal{T} \cap H_{P}^{1}$, and the strong convergence in $X_{P}$, uniformly with respect to $r \in \mathbb{R}$

$$
\lim _{S \rightarrow+\infty} \frac{1}{S} \int_{r}^{r+S} \nabla u_{s} \mathrm{~d} s=\nabla\langle u\rangle
$$

For the last statement use $\left|\nabla u_{s}\right|_{X_{P}}=|\nabla u|_{X_{P}}, s \in \mathbb{R}$ and the above convergence.

Proof. (of Lemma 2.1)

We proceed in two steps.

1. Assume for the moment that $D \in H_{Q} \cap H_{Q}^{\infty}$. We know by Theorem 2.2 that

$$
\lim _{S \rightarrow+\infty} \frac{1}{S} \int_{r}^{r+S} G(s) D \mathrm{~d} s=\langle D\rangle \text { strongly in } H_{Q}, \text { uniformly with respect to } r \in \mathbb{R} .
$$

We define the sequence $c_{k}=\mathbf{1}_{\left\{\left|P^{1 / 2} c\right| \leq k\right\}} c, k \in \mathbb{N}$. Any vector field $c_{k}$ belongs to $X_{P}^{\infty}$ and we have the convergence $\lim _{k \rightarrow+\infty} c_{k}=c$ in $X_{P}$. For any $k \in \mathbb{N}$ we have $\lim _{S \rightarrow+\infty} \frac{1}{S} \int_{r}^{r+S} G(s) D c_{k} \mathrm{~d} s=\langle D\rangle c_{k}$, strongly in $X_{Q}$, uniformly with respect to $r \in \mathbb{R}$ thanks to the inequality

$$
\left|\frac{1}{S} \int_{r}^{r+S} G(s) D c_{k} \mathrm{~d} s-\langle D\rangle c_{k}\right|_{X_{Q}} \leq\left|\frac{1}{S} \int_{r}^{r+S} G(s) D \mathrm{~d} s-\langle D\rangle\right|_{H_{Q}}\left|c_{k}\right|_{X_{P}^{\infty}} .
$$


Observe that

$$
\begin{aligned}
\left|\frac{1}{S} \int_{r}^{r+S} G(s) D c \mathrm{~d} s-\langle D\rangle c\right|_{X_{Q}} & \leq\left|\frac{1}{S} \int_{r}^{r+S} G(s) D\left(c-c_{k}\right) \mathrm{d} s\right|_{X_{Q}} \\
& +\left|\frac{1}{S} \int_{r}^{r+S} G(s) D c_{k} \mathrm{~d} s-\langle D\rangle c_{k}\right|_{X_{Q}}+\left|\langle D\rangle\left(c_{k}-c\right)\right|_{X_{Q}} \\
& \leq\left|\frac{1}{S} \int_{r}^{r+S} G(s) D \mathrm{~d} s\right|_{H_{Q}^{\infty}}\left|c-c_{k}\right|_{X_{P}} \\
& +\left|\frac{1}{S} \int_{r}^{r+S} G(s) D c_{k} \mathrm{~d} s-\langle D\rangle c_{k}\right|_{X_{Q}}+|\langle D\rangle|_{H_{Q}^{\infty}}\left|c_{k}-c\right|_{X_{P}} \\
& \leq 2|D|_{H_{Q}^{\infty}}\left|c_{k}-c\right|_{X_{P}}+\left|\frac{1}{S} \int_{r}^{r+S} G(s) D c_{k} \mathrm{~d} s-\langle D\rangle c_{k}\right|_{X_{Q}}
\end{aligned}
$$

which implies that for any $k \in \mathbb{N}$

$$
\limsup _{S \rightarrow+\infty} \sup _{r \in \mathbb{R}}\left|\frac{1}{S} \int_{r}^{r+S} G(s) D c \mathrm{~d} s-\langle D\rangle c\right|_{X_{Q}} \leq 2|D|_{H_{Q}^{\infty}}\left|c_{k}-c\right|_{X_{P}} .
$$

Our conclusion follows by letting $k \rightarrow+\infty$.

2. Assume now that $D \in H_{Q}^{\infty}$. For any $k \in \mathbb{N}$ we consider $D_{k}=1_{\{\psi \leq k\}} D$. Since $D \in H_{Q}^{\infty} \subset H_{Q \text {,loc }}$, we deduce that $D_{k} \in H_{Q} \cap H_{Q}^{\infty}$, and by the previous statement, we have for any $k \in \mathbb{N}$

$$
\lim _{S \rightarrow+\infty} \sup _{r \in \mathbb{R}}\left|\frac{1}{S} \int_{r}^{r+S} G(s) D_{k} c \mathrm{~d} s-\left\langle D_{k}\right\rangle c\right|_{X_{Q}}=0 .
$$

Observe that

$$
\begin{aligned}
\left|\frac{1}{S} \int_{r}^{r+S} G(s) D c \mathrm{~d} s-\langle D\rangle c\right|_{X_{Q}} & -\left|\frac{1}{S} \int_{r}^{r+S} G(s) D_{k} c \mathrm{~d} s-\left\langle D_{k}\right\rangle c\right|_{X_{Q}} \leq\left|\frac{1}{S} \int_{r}^{r+S} G(s)\left(D-D_{k}\right) c \mathrm{~d} s\right|_{X_{Q}} \\
& +\left|\left\langle D_{k}-D\right\rangle c\right|_{X_{Q}} \\
& =\left|\frac{1}{S} \int_{r}^{r+S} G(s) D \mathbf{1}_{\{\psi>k\}} c \mathrm{~d} s\right|_{X_{Q}}+\left|\langle D\rangle \mathbf{1}_{\{\psi>k\}} c\right|_{X_{Q}} \\
& \leq \frac{1}{S} \int_{r}^{r+S}|G(s) D|_{H_{Q}^{\infty}}\left|\mathbf{1}_{\{\psi>k\}} c\right|_{X_{P}} \mathrm{~d} s+|\langle D\rangle|_{H_{Q}^{\infty}}\left|\mathbf{1}_{\{\psi>k\}} c\right|_{X_{P}} \\
& \leq 2|D|_{H_{Q}^{\infty}}\left|\mathbf{1}_{\{\psi>k\}} c\right|_{X_{P}}
\end{aligned}
$$

which implies that

$$
\limsup _{S \rightarrow+\infty} \sup _{r \in \mathbb{R}}\left|\frac{1}{S} \int_{r}^{r+S} G(s) D c \mathrm{~d} s-\langle D\rangle c\right|_{X_{Q}} \leq 2|D|_{H_{Q}^{\infty}}\left|\mathbf{1}_{\{\psi>k\}} c\right|_{X_{P}} .
$$

Our conclusion follows by letting $k \rightarrow+\infty$. 


\section{Acknowledgments}

This work has been carried out within the framework of the French Federation for Magnetic Fusion Studies (FR-FCM) and of the Eurofusion consortium, and has received funding from the Euratom research and training programme 2014-2018 and 2019-2020 under grant agreement No 633053. The views and opinions expressed herein do not necessarily reflect those of the European Commission.

\section{References}

[1] T. Blanc, Numerical methods for computing an averaged matrix field. Application to the asymptotic analysis of a parabolic problem with stiff transport terms, SIAM J. Multiscale Model. Simul. 17(2019) 531-551.

[2] T. Blanc, M. Bostan, Multi-scale analysis for highly anisotropic parabolic problems, Discrete Contin. Dyn. Syst. Ser. B 25(2020) 335-399.

[3] T. Blanc, M. Bostan, F. Boyer, Asymptotic analysis of parabolic equations with stiff transport terms by a multi-scale approach, Discrete Contin. Dyn. Syst. Ser. A 37(2017) 4637-4676.

[4] M. Bostan, Transport equations with disparate advection fields. Application to the gyrokinetic models in plasma physics, J. Differential Equations 249(2010) 1620-1663.

[5] M. Bostan, Strongly anisotropic diffusion problems; asymptotic analysis, J. Differential Equations 256(2016) 1043-1092.

[6] M. Bostan, Multi-scale analysis for linear first order PDEs. The finite Larmor radius regime, SIAM J. Math. Anal. 48(2016) 2133-2188.

[7] S. Britt, S. Tsynkov, E. Turkel, Numerical solution of the wave equation with variable wave speed on non conforming domains by high-order differential potentials, J. Comput. Phys. 354(2018) 26-42.

[8] R. Dautray, J.-L. Lions, Analyse mathématique et calcul numérique pour les sciences et les techniques, vol. 8, Masson 1988.

[9] P. Degond, F. Deluzet, C. Negulescu, An asymptotic preserving scheme for strongly anisotropic elliptic problems, Multiscale Model. Simul. 8(2009/10) 645666.

[10] L. Fezoui, S. Lanteri, S. Lohrengel, S. Piperno, Convergence and stability of a discontinuous Galerkin time-domain method for the 3D heterogeneous Maxwell equations on unstructured meshes, M2AN Math. Model. Numer. Anal. 39(2005) 1149-1176.

[11] F. Filbet, C. Negulescu, C. Yang, Numerical study of a nonlinear heat equation for plasma physics, Int. J. Comput. Math. 89(2012) 1060-1082. 
[12] J.C. Gilbert, P. Joly, Higher order time stepping for second order hyperbolic problems and optimal cfl conditions, Partial Differential Equations, Modeling and Numerical Simulation, Comput. Meth. Appl. Sci. 16(2006) 67-93.

[13] S. Jin, Asymptotic preserving schemes for multi scale kinetic and hyperbolic equations : a review, Riv. Mat. Univ. Parma 3(2012) 177-216.

[14] J.-L. Lions, E. Magenes, Non-homogeneous boundary value problems and applications, vol. I, Springer Berlin Heidelberg, 1972.

[15] M. Reed, B. Simon, Methods of Modern Mathematical Physics, vol. I, Functional Analysis, Academic Press, 1980.

[16] P. Sharma, G.W. Hammett, Preserving monotonicity in anisotropic diffusion, J. Comput. Phys. 227(2007) 123-142.

[17] P. Sharma, G.W. Hammett, A fast semi-implicit method for anisotropic diffusion, J. Comput. Phys. 230(2011) 4899-4909. 